ARTICLE

DOI: $10.1038 /$ s41467-018-05771-8

\title{
Galectin-9 binds IgM-BCR to regulate B cell signaling
}

Anh Cao (1) ${ }^{1}$, Nouf Alluqmani ${ }^{2}$, Fatima Hifza Mohammed Buhari ${ }^{3}$, Laabiah Wasim', Logan K. Smith', Andrew T. Quaile (i) ${ }^{4}$, Michael Shannon ${ }^{5}$, Zaki Hakim², Hossai Furmli ${ }^{3}$, Dylan M. Owen ${ }^{5}$, Alexei Savchenko ${ }^{4,6}$ \& Bebhinn Treanor (1) 1,2,3

The galectin family of secreted lectins have emerged as important regulators of immune cell function; however, their role in B-cell responses is poorly understood. Here we identify $\operatorname{lgM}-\mathrm{BCR}$ as a ligand for galectin-9. Furthermore, we show enhanced BCR microcluster formation and signaling in galectin-9-deficient B cells. Notably, treatment with exogenous recombinant galectin-9 nearly completely abolishes BCR signaling. We investigated the molecular mechanism for galectin-9-mediated inhibition of BCR signaling using superresolution imaging and single-particle tracking. We show that galectin-9 merges pre-existing nanoclusters of $I g M-B C R$, immobilizes IgM-BCR, and relocalizes IgM-BCR together with the inhibitory molecules CD45 and CD22. In resting naive cells, we use dual-color superresolution imaging to demonstrate that galectin- 9 mediates the close association of $\operatorname{lgM}$ and CD22, and propose that the loss of this association provides a mechanism for enhanced activation of galectin-9-deficient B cells.

\footnotetext{
${ }^{1}$ Department of Immunology, University of Toronto, 1 King's College Circle, Toronto, ON M5S 1A8, Canada. ${ }^{2}$ Department of Cell and Systems Biology, University of Toronto, 24 Harbord Street, Toronto, ON M5S 3G5, Canada. ${ }^{3}$ Department of Biological Sciences, University of Toronto Scarborough, 1265 Military Trail, Toronto, ON M1C 1A4, Canada. ${ }^{4}$ Department of Chemical Engineering and Applied Chemistry, University of Toronto, 200 College Street, Toronto, ON M5S 3E5, Canada. ${ }^{5}$ Department of Physics and Randall Division of Cell and Molecular Biophysics, New Hunt's House, King's College London Guy's Campus, London SE1 1UL, UK. ${ }^{6}$ Department of Microbiology, Immunology and Infectious Diseases, Cumming School of Medicine, Health Research Innovation Centre 4AA06, University of Calgary, 3330 Hospital Drive NW, Calgary, AB T2N 4N1, Canada. These authors contributed equally: Anh Cao, Nouf Alluqmani, Fatima Hifza Mohammed Buhari, Laabiah Wasim. Correspondence and requests for materials should be addressed to B.T. (eamil: bebhinn.treanor@utoronto.ca)
} 
$\mathrm{B}$ cells play a critical role in the immune response and production of protective antibodies. B-cell activation is triggered by binding of antigen to the B-cell receptor (BCR), which initiates a cascade of intracellular signaling through assembly of a multiprotein complex of kinases and adaptors ${ }^{1}$. B-cell activation is accompanied by formation of numerous signaling microclusters ${ }^{2}$. Similar microstructures of antigen receptors have been described in $\mathrm{T}$ cells ${ }^{3}$ and thus have been proposed to represent the basic unit of lymphocyte signaling ${ }^{4}$. These observations implicate receptor clustering as a mechanism to regulate signaling events, and consequently the cellular outcome of receptor engagement. Indeed, the size and spatial patterning of signaling assemblies significantly contribute to cellular outcomes, with even small variations resulting in altered responses ${ }^{5-7}$.

Two key parameters influencing the assembly of signaling clusters and regulation of membrane receptor activation are the constitutive nanoscale clustering of membrane proteins referred to as nanoclusters or protein islands ${ }^{8-10}$, and the cell surface mobility of membrane proteins (or nanoclusters of proteins) $7,11,12$. These parameters have important implications for receptor triggering and the assembly of signaling complexes as they influence the interaction between protein partners. Several mechanisms have been identified that impact on the organization and mobility of membrane proteins, including the actin cytoskeleton $^{11-13}$, protein-protein interactions ${ }^{9,14-16}$, and membrane microdomains defined by lipid composition ${ }^{8,17}$.

An often overlooked mechanism controlling membrane protein organization and mobility is the interaction of these cell surface glycoproteins with the family of soluble secreted lectins, known as galectins, which bind and crosslink cell surface proteins, generating glycan-based domains ${ }^{18}$. Indeed, the galectin lattice influences glycoprotein compartmentalization and lateral mobility at the cell surface ${ }^{19-21}$. These proteins have emerged as important regulators of the immune response. For example, $\mathrm{T}$ cells from mice deficient in Mgat5, which encodes a glycosyltransferase involved in generation of galectin-binding epitopes, have enhanced T-cell receptor (TCR) clustering at the immunological synapse and increased TCR signaling ${ }^{22}$. In comparison to $\mathrm{T}$ cells, relatively little is known about glycan-galectin interactions in B cells; however, pre-BCR clustering at the pre-B-cellstromal cell synapse is dependent on pre-BCR/galectin-1/integrin interactions ${ }^{23}$. Interestingly, a recent study demonstrated decreased antibody titers upon immunization in mice treated with recombinant galectin- $9^{24}$. Galectin- 9 belongs to the tandemrepeat subfamily of galectins, which contain two different carbohydrate recognition domains (CRDs) separated by a flexible linker ${ }^{25}$. In T cells, galectin-9 induces cell death of T helper type 1 $\left(\mathrm{T}_{\mathrm{H}} 1\right)$ cells through binding to Tim- $3^{26}$, as well as suppresses generation of $\mathrm{T}_{\mathrm{H}} 17$ cells and promotes induction of $\mathrm{T}_{\text {regs }} 27$. However, the role of galectin- 9 in B cells, and the molecular mechanism for decreased antibody production in galectin-9treated mice has not been investigated.

Here we identify IgM-BCR and CD45 as ligands for galectin-9. Further, we show enhanced BCR microcluster formation and signaling in galectin-9-deficient B cells. Notably, treatment with exogenous recombinant galectin-9 (rGal9) nearly completely abolishes BCR signaling. We investigated the molecular mechanism for galectin-9-mediated inhibition of BCR signaling. We show using super-resolution imaging and single-particle tracking that galectin-9 brings together pre-formed BCR nanoclusters and reduces BCR mobility at the cell surface, and consequently attenuates BCR microcluster formation. We further interrogated the effect of galectin-9 on the organization of cell surface proteins and demonstrate that galectin-9 increases the molecular density of IgM, CD45, CD22, and CD19 within this galectin lattice. Using dual-color direct stochastic optical reconstruction microscopy (dSTORM), we show that galectin-9 mediates close association of IgM and CD22 in resting naive B cells and thus propose a novel role for galectin- 9 in regulation of B-cell activation.

\section{Results}

Galectin-9 is bound to the surface of primary naive B cells. To investigate the underlying mechanism for decreased antibody production in galectin-9-treated mice $^{24}$, we initially asked if galectin-9 is bound to the surface of B cells. Primary naive B cells were isolated from $\mathrm{C} 57 \mathrm{BL} / 6$ (wild type; WT) and Lgals9-/(Gal9-KO) mice, stained with a fluorescently labeled antibody specific for galectin-9 and examined by flow cytometry and confocal microscopy. We found that galectin-9 is bound to the surface of WT B cells (Fig. 1a), organized in discrete puncta (Fig. 1b). To investigate the in vivo expression of galectin-9, we immunostained inguinal lymph nodes to identify subcapsular sinus macrophages (CD169), B cells (B220), and galectin-9. We found that galectin-9 was readily detectable within the B-cell follicle (Fig. 1c).

Galectin-9 regulates BCR microclusters and signaling. To investigate the effect of galectin-9 deficiency on BCR microcluster formation we employed artificial planar lipid bilayers in which fluorescently labeled anti-BCR antibodies as surrogate antigen are tethered to mimic the in vivo environment of antigen encoun$\operatorname{ter}^{28}$. Naive B cells from WT and Gal9-KO mice were settled on lipid bilayers containing anti-kappa, fixed after $90 \mathrm{~s}$, and visualized by confocal microscopy. WT B cells spread and form multiple BCR-antigen microclusters ${ }^{2,29}$ (Fig. 2a). Gal9-KO B cells also spread and form microclusters; however, the area of cellbilayer contact, and total intensity of antigen is increased (Fig. 2a-c). Moreover, the mean antigen intensity is also increased in Gal9-KO B cells, indicating that the amount of BCR-antigen within individual clusters is increased (Fig. 2d). Given this enhanced microcluster formation, we asked if BCR signaling was altered in Gal9-deficient B cells. Consistent with microcluster data, total tyrosine phosphorylation was increased in Gal9-KO B cells (Fig. 2e). To further investigate this enhanced signaling, we examined phosphorylation of downstream signaling molecules, including CD19, Akt, and extracellular regulated kinase $($ ERK1/2). Although we did not detect any increase in phosphoCD19 or phospho-Akt, we found that phosphorylation of ERK1/2 was increased in Gal9-KO B cells (Fig. $2 \mathrm{f}-\mathrm{h}$ ). We verified that these differences were not due to altered expression of BCR or CD19 (Supplementary Fig. 1). These data demonstrate that galectin-9 regulates BCR microcluster formation and signaling.

To determine if enhanced microcluster formation and signaling is specifically due to loss of galectin-9 at the cell surface, we titrated the amount of recombinant galectin- 9 ( $\mathrm{rGal} 9$ ) added to Gal9-KO B cells from 0.1 to $1 \mu \mathrm{M}$ and then stained cells with anti-galectin-9 antibody and compared this to untreated WT cells. We found that $0.1 \mu \mathrm{M}$ rGal9 added to Gal9-KO cells was roughly equivalent to the level of endogenous galectin-9 on WT cells (Fig. 3a). We treated Gal9-KO B cells with $0.1 \mu \mathrm{M}$ rGal9 and then stimulated with anti-IgM and examined ERK phosphorylation as we found this pathway was enhanced in galectin9-deficient cells. We found that this treatment was sufficient to restore BCR signaling to that observed in WT B cells (Fig. 3b), demonstrating that the effect of deficiency of galectin-9 is indeed due to lack of galectin-9 at the cell surface. Given that galectin family members share the basic glycan ligand, $N$-acetyllactosamine, we examined an alternative galectin-deficient mouse model. In contrast to Gal9-KO B cells, we found no difference in BCR 
a

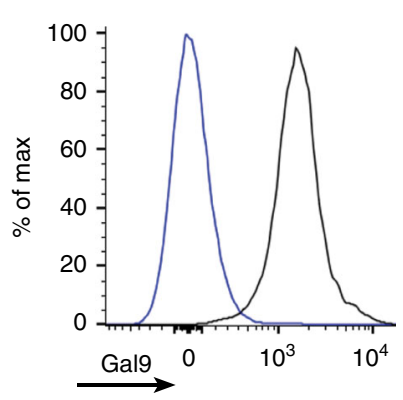

b

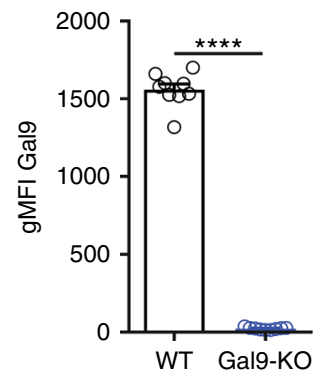

DIC

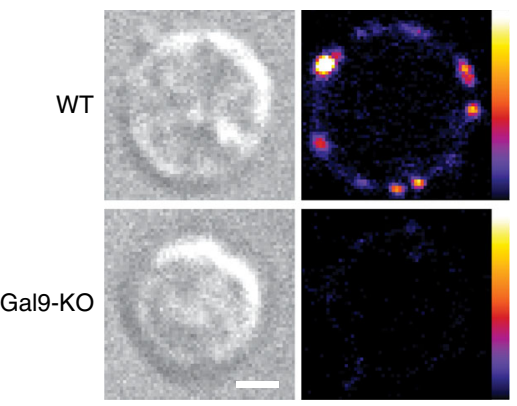

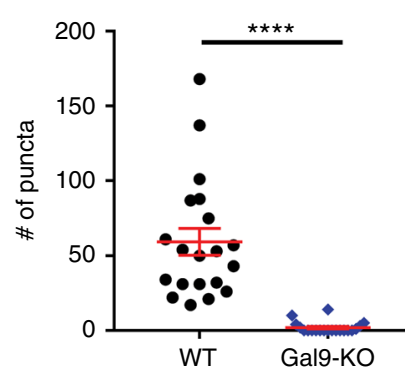

C

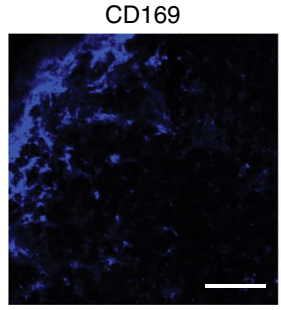

B220

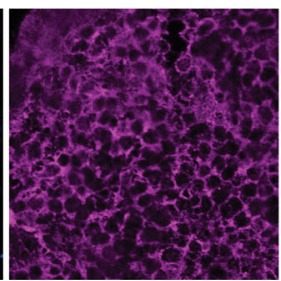

Gal9

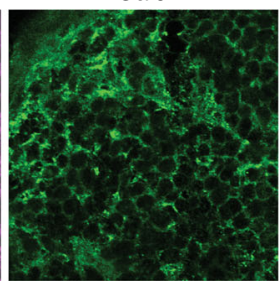

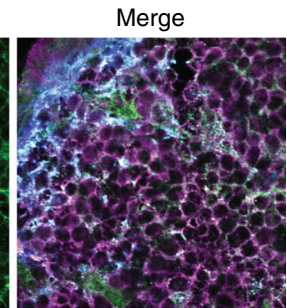

Fig. 1 Galectin-9 is bound to the surface of primary naive B cells. a Representative flow cytometry plot (left) and quantification (right) of geometric mean \pm SEM of surface staining for galectin-9 in WT (black) and Gal9-KO (blue) B cells from nine independent experiments. b Representative DIC (left) and confocal microscopy images (right) mapped to an 8-bit fire color scale (ImageJ) of primary WT (top) and Gal9-KO B cells (bottom) stained for surface galectin-9. Quantification of number of galectin-9 puncta is shown on the right (each dot represents 1 cell, 20 cells measured per condition) with the mean \pm SEM indicated by the red bar. Scale bar $2 \mu \mathrm{m}$. Data representative of three independent experiments. c Representative confocal microscopy images of cryosections of the inguinal lymph node of WT B cells stained for subcapsular sinus macrophages (CD169; blue), B cells (B220; magenta), and Gal9 (green). Scale bar $20 \mu \mathrm{m}$. Data representative of three independent experiments. Statistical significance was assessed by Mann-Whitney, ${ }^{\star \star \star \star} p<0.0001$

microcluster formation or downstream signaling in galectin-1deficient B cells (Supplementary Fig. 2). These data, while not exhaustive of the galectin family, provide strong evidence for specificity of galectin-9 in regulation of these early events of B-cell activation, consistent with studies demonstrating that structural differences in the carbohydrate recognition domain (CRD) of galectin-1 and galectin-9 define specific interactions with cell surface glycoproteins ${ }^{30}$.

We noted that ligands for galectin- 9 at the cell surface were not saturated in primary naive B cells and we could add 10-fold more rGal9 to both WT and Gal9-KO B cells (Fig. 3a). Thus, we asked what happens to BCR signaling if we increase the amount of galectin-9 bound to the surface. We observed a substantial reduction in total tyrosine phosphorylation, as well as phosphorylation of CD19, Akt, and ERK upon pre-treatment of WT cells with rGal9 (Fig. 3c-f). These differences were not due to altered expression of BCR, CD19, or CD45 by rGal9 (Supplementary Fig. 3), nor galectin-9-induced cell death (Supplementary Fig. 4), as has been reported for thymocytes and $\mathrm{T}$ cells ${ }^{26,31,32}$.

Galectin-9 binds IgM-BCR and CD45. Our data demonstrate an important role for galectin-9 in regulating BCR signaling; however, the cell surface glycoproteins that bind galectin-9 in B cells have not been identified. To identify potential ligands we performed a pull-down assay using rGal9 followed by mass spectrometry ${ }^{33}$. To not compete with endogenous galectin-9, Gal9-KO primary B cells were lysed, incubated with FLAG-tagged rGal9 followed by magnetic separation using beads conjugated with anti-FLAG, and the ligands were eluted using lactose (Fig. 4a). Isolated proteins were subjected to in-solution tryptic digestion and liquid chromatography tandem mass spectrometry. We identified several potential ligands of galectin-9 in B cells, including CD45 (Ptprc), IgM-BCR (Ighm), and the BCRassociated signaling chain CD79b ( $\operatorname{Ig} \beta$ ) (Table 1). To confirm the identity of these ligands, we performed a pull-down assay using rGal9 under stringent cell lysis conditions to disrupt protein-protein interactions followed by western blotting (Fig. 4b). Consistent with our mass spectrometry results, we identified CD45 and IgM-BCR as potential ligands of galectin-9. To verify that these were indeed direct ligands of galectin-9, we performed far-western analysis ${ }^{34}$ using FLAG-tagged rGal9 as bait protein. We detected galectin-9-binding proteins corresponding to the size of CD45 and IgM in reference blots (Fig. 4c) in primary murine $\mathrm{B}$ cells. Importantly, a band corresponding to the size of IgM was not detected in far-western using cell lysates from the A20 B-cell line that does not express endogenous IgM. Conversely, a band corresponding to the size of IgM was detected in lysates of A20 B cells expressing hen egg lysozyme (Hel)specific IgM (D1.3). Neither CD45 (B220) nor IgM bands were detected when Jurkat $\mathrm{T}$ cells were used. These data provide strong evidence that galectin-9 binds IgM-BCR and CD45.

Galectin-9 alters IgM-BCR nanoclusters. Identification of IgMBCR as a ligand of galectin-9, and the potential of galectins to act as cell surface scaffolding proteins ${ }^{19}$ led us to ask whether galectin-9 has a role in organizing IgM-BCR nanoclusters. To investigate this, we employed dSTORM ${ }^{35}$, which permits a lateral resolution of approximately $20 \mathrm{~nm}$, and has been used to study the organization of $\operatorname{IgM}$, IgD, and $\operatorname{IgG} \mathrm{BCRs}^{9,10,36}$. Primary WT and Gal9-KO B cells were labeled with Alexa Fluor 647-conjugated anti-IgM Fab fragment, settled on non-BCR-stimulating anti-major histocompatibility complex (MHC) II-coated coverslips to adhere cells, then fixed and imaged by total internal reflection fluorescence microscopy (TIRFM). IgM-BCR are organized into nanoscale clusters in WT B cells ${ }^{9}$ (Fig. 5a). Nanoclusters of IgM-BCR were also clearly visible in dSTORM images in Gal9-KO B cells, with no obvious difference compared to WT B cells (Fig. 5a). To quantify clustering tendency of IgM-BCR, we used two methods commonly used to assess distribution of proteins from super-resolution images ${ }^{37}$. The 
a

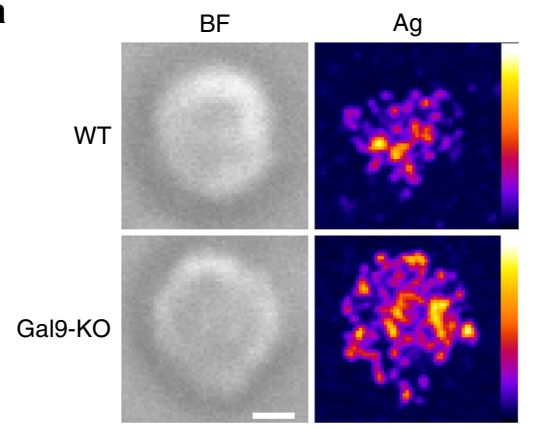

b

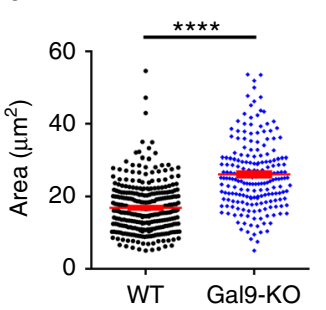

c

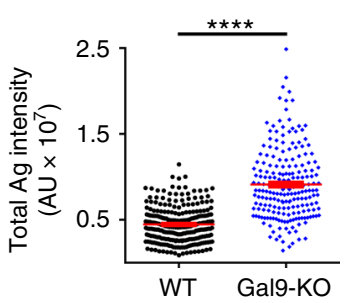

d

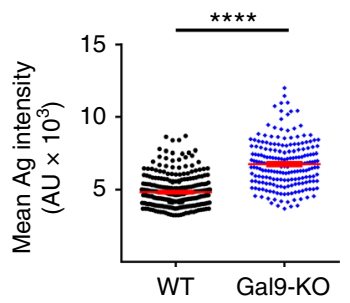

e

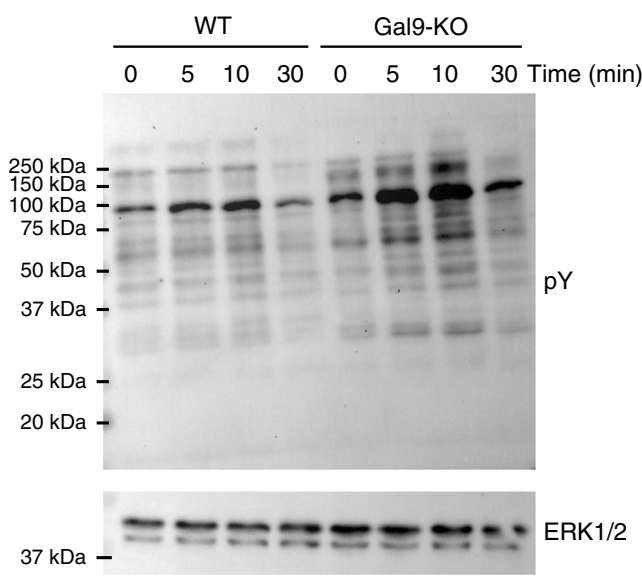

f
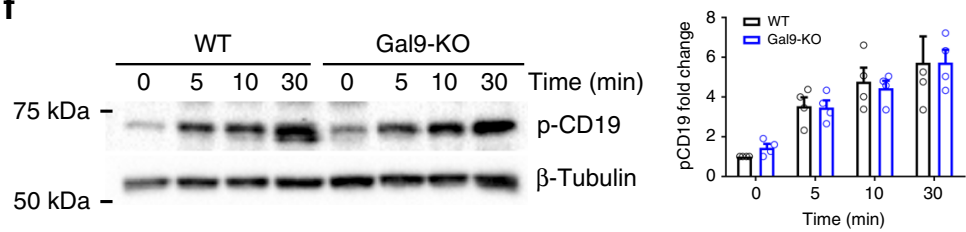

g
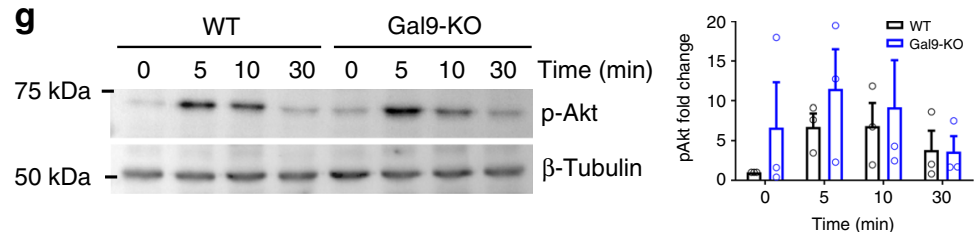

h

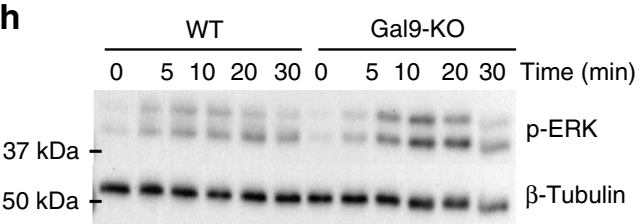

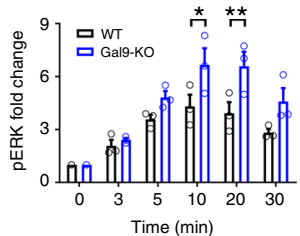

Fig. 2 Galectin-9 regulates BCR microcluster formation and signaling. a Representative images of primary naive WT (top) and Gal9-KO B cells (bottom) fixed on bilayers containing anti-kappa as surrogate antigen $(\mathrm{Ag})$ after $90 \mathrm{~s}$ of spreading and imaged by confocal microscopy. Brightfield (left) and confocal (right) visualizing antigen mapped to an 8-bit fire color scale (ImageJ). Scale bar $2 \mu \mathrm{m}$. Quantification of $\mathbf{b}$ area of spreading, $\mathbf{c}$ total antigen fluorescence intensity at the cell-bilayer contact, and $\mathbf{d}$ mean intensity of antigen for WT (black circles) and Gal9-KO (blue diamonds) cells (each dot represents 1 cell, 200 cells measured per condition), with the mean \pm SEM indicated by the red bar, ${ }^{\star \star \star \star} p<0.0001$, Mann-Whitney test. Data representative of at least three independent experiments. e-h Primary naive B cells from WT and Gal9-KO mice were settled onto anti-lgM-coated plates for the indicated time. Cells were lysed and subjected to SDS-PAGE followed by immunoblotting with e anti-phosphotyrosine and anti-ERK1/2, f anti-phospho-CD19, $\mathbf{g}$ anti-phosphoAkt, and $\mathbf{h}$ anti-phospho ERK1/2 (pERK) and anti- $\beta$ tubulin. Data representative of at least three independent experiments. $\mathbf{f}$ Quantification of the fold increase in pCD19, pAkt, and pERK, with the mean \pm SEM indicated by bar. Data were analyzed by two-way ANOVA, followed by Sidak's multiple comparisons test; ${ }^{\star} p<0.05,{ }^{\star \star} p<0.01$

Hopkins index, which evaluates clustering tendency compared to a random distribution (value of 0.5 ), was 0.75 for IgM-BCR on both WT and Gal9-KO B cells (Fig. 5b). The $H$ function derived from Ripley's $K$ function evaluates the extent of clustering; distance of the $H$ function peak is related to cluster radius and peak height depends on density of molecules in clusters. We found no difference in the $H$ function curve in Gal9-KO B cells compared to WT B cells. These findings suggest that galectin-9 does not mediate formation of IgM-BCR nanoclusters; however, galectin-9 is sparsely distributed at the cell surface of naive B cells (Fig. 1b), and therefore dSTORM analysis based on randomly selected regions may underestimate an effect specifically within the galectin-9 lattice. To focus our dSTORM analysis on the galectin9 lattice, we treated Gal9-KO B cells with fluorescently labeled rGal9. We found that this treatment altered the organization of IgM-BCR, which appeared more clustered compared to WT and Gal9-KO cells (Fig. 5a). Both the Hopkin's index and the $H$ function of Ripley's $K$ derived from regions where galectin-9 localized indicated that IgM-BCR was more highly clustered (Fig. 5b, c). The radius of clusters of IgM-BCR inside the galectin9 lattice is approximately $150-250 \mathrm{~nm}$, compared to $50-100 \mathrm{~nm}$ in WT and Gal9-KO B cells (Fig. 5c). To further quantify the effect of galectin-9 on organization of IgM, we used Bayesian cluster analysis ${ }^{38}$. This method is advantageous because: (1) it accounts for non-negligible unclustered background, which can interfere with Ripley's and Hopkins analysis; (2) it takes account of the uncertainty associated with each localization assigned by the Gaussian function; and (3) it reduces the bias arising from subjective user-defined analysis parameters, such as threshold and cluster radius. Using this method, we identified the number of clusters per region, cluster radii, number of molecules per cluster, and percentage of localizations in clusters from $3 \times 3 \mu \mathrm{m}$ regions of interest from reconstructed images. Treatment with rGal9 decreased the number of clusters (Fig. 5d), whereas radius of clusters (Fig. 5e) and number of molecules per cluster (Fig. 5f) increased. There was no difference in percentage of localizations within clusters between WT, Gal9$\mathrm{KO}$, and Gal9-KO treated with rGal9 (Fig. 5g). These findings suggest that galectin-9 brings pre-existing IgM-BCR nanoclusters together, increasing size of clusters and number of molecules per cluster, but without affecting percentage of molecules residing in clusters. 
a

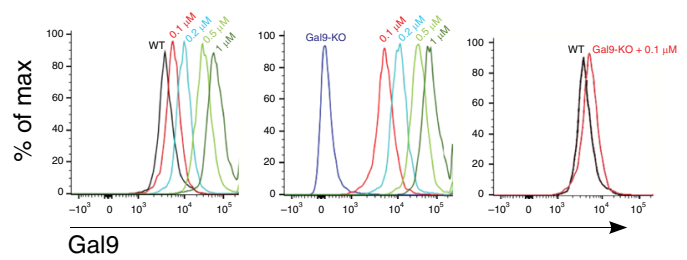

C

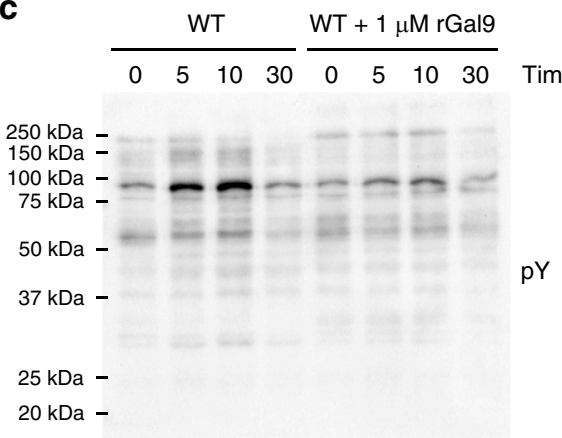

$50 \mathrm{kDa}-\longrightarrow-\ldots-$-Tubulin b
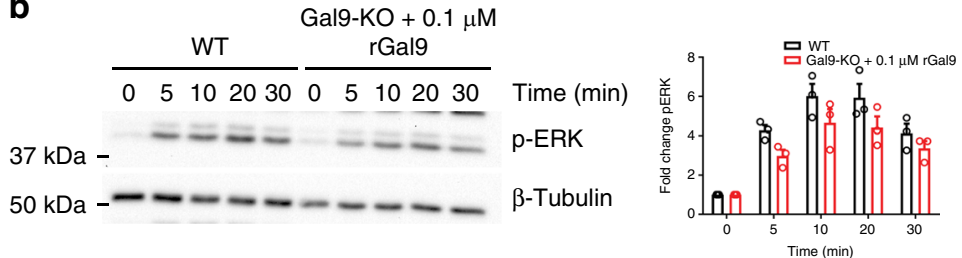

d
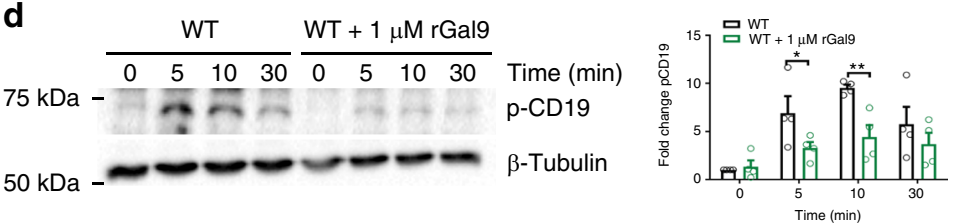

e
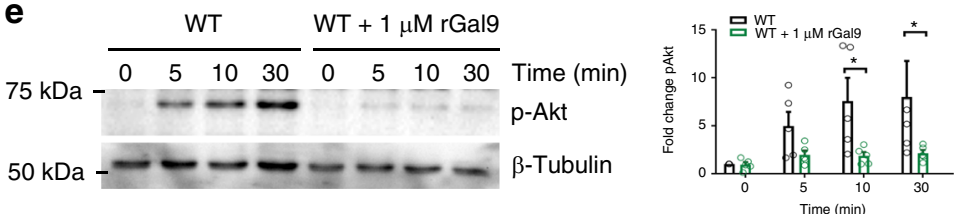

$\mathbf{f}$

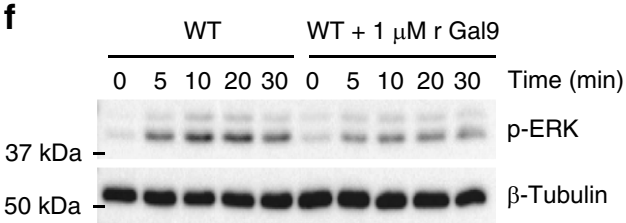

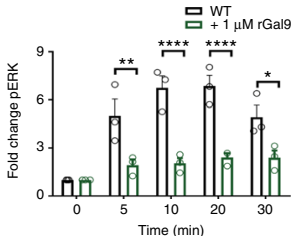

Fig. 3 Treatment with exogenous galectin-9 suppresses BCR signaling. a Representative flow cytometric histograms of WT (left) and Gal9-KO (middle) $B$ cells either untreated or treated with various concentrations of recombinant galectin-9 ( $r$ Gal9; $0.1,0.2,0.5$, and $1 \mu \mathrm{M}$ ) followed by surface staining for galectin-9 and analyzed using flow cytometry. Overlay of endogenous galectin-9 surface expression in WT cells, and Gal9-KO cells treated with $0.1 \mu \mathrm{M}$ rGal9 (right). b Naive B cells from WT and Gal9-KO mice treated with $0.1 \mu \mathrm{M}$ rGal9 were settled onto anti-lgM-coated plates for the indicated time. Cells were lysed and subjected to SDS-PAGE followed by immunoblotting with anti-phospho ERK1/2 and anti- $\beta$ tubulin (left panel). Quantification of the fold change in pERK over time, averaged over two independent experiments with the mean \pm SEM indicated by the bar (right panel). c-f Naive B cells from WT mice were treated with $1 \mu \mathrm{M} \mathrm{rGal9}$ and settled onto anti-lgM-coated plates for the indicated time. Cells were lysed and subjected to SDS-PAGE followed by immunoblotting with $\mathbf{c}$ anti-phosphotyrosine and ERK1/2, d anti-phospho-CD19, e anti-phospho-Akt, and $\mathbf{f}$ anti-phospho ERK1/2 and anti- $\beta$ tubulin. Quantification of the fold change in pCD19, pAkt, and pERK over time, averaged over three independent experiments, with the mean \pm SEM indicated by the bar is shown in the right panel. Statistical significance measure by two-way ANOVA followed by Sidak's multiple comparisons test; ${ }^{{ }^{\star \star \star \star}} p<0.0001,{ }^{\star \star} p<$ $0.01,{ }^{*} p<0.05$

Galectin-9 immobilizes IgM-BCR. The mobility of IgM-BCR is a critical factor regulating BCR signaling 9,12 . Given dSTORM analysis showed galectin-9 increased cluster size, we wondered whether altered BCR mobility may account for suppressed BCR signaling upon treatment with $\mathrm{rGal}$ 9. To investigate the effect of galectin-9 on IgM mobility in the steady state, we labeled single particles of IgM on WT and Gal9-KO B cells with Atto-633conjugated anti-IgM Fab fragments. Cells were settled on antiMHC II-coated coverslips and single molecules of IgM were visualized using TIRFM. The median diffusion coefficient of IgMBCR on Gal9-KO B cells was approximately $30 \%$ higher than that on WT B cells $\left(0.037 \mu \mathrm{m}^{2} / \mathrm{s}\right.$ compared to $0.027 \mu \mathrm{m}^{2} / \mathrm{s}$; Fig. $\left.6 \mathrm{a}\right)$, consistent with a decrease in the relative frequency of slowmoving IgM-BCR in Gal9-KO B cells (Fig. 6b). To examine the mobility of IgM-BCR inside the galectin- 9 lattice, we treated Gal9-KO B cells with fluorescently labeled rGal9 and visualized and tracked IgM-BCR. We defined regions of high and low galectin-9 using the fluorescence intensity of galectin- 9 and then calculated diffusion coefficient in these regions (Fig. 6c). The median diffusion coefficient of IgM in galectin-9-high regions was reduced twofold compared to galectin-9-low regions $\left(0.012 \mu \mathrm{m}^{2} / \mathrm{s}\right.$ compared to $0.028 \mu \mathrm{m}^{2} / \mathrm{s}$ ) (Fig. 6d). Approximately $65 \%$ of tracked IgM molecules are largely immobilized inside the mask compared to $45 \%$ of tracked IgM molecules outside the mask (Fig. 6e). This finding provides direct evidence for galectin-9 to restrict the mobility of IgM molecules on the B-cell membrane.

We hypothesized that galectin-9-mediated immobilization of IgM-BCR would reduce BCR localization to the contact with an antigen-containing membrane. To test this, we settled WT B cells or cells pre-treated with rGal9 on planar lipid bilayers containing anti-kappa as surrogate antigen for $90 \mathrm{~s}$ and then fixed and visualized by confocal microscopy. We found that BCR-antigen aggregation is reduced in cells treated with rGal9 (Fig. 6f, g). These findings suggest that galectin-9 binds IgM-BCR inducing aggregation and immobilization of IgM nanoclusters, which consequently affects the formation of signaling microclusters upon BCR stimulation.

Galectin-9 increases the density of IgM-BCR and co-receptors. Our finding that rGal9 induces an increase in the size of IgMBCR clusters and immobilization of IgM are also features consistent with the activation of $\mathrm{B}$ cells ${ }^{9,10,15,39}$. To examine if galectin-9 mediated clustering of $\operatorname{IgM}$ was sufficient to induce signaling, we examined calcium flux upon treatment of cells with rGal9 by flow cytometry. In contrast to stimulation with antiBCR antibodies, we did not detect any increase in intracellular 
a Potential ligand(s) Flag Flag-tagged galectin-9 " Anti-flag antibody

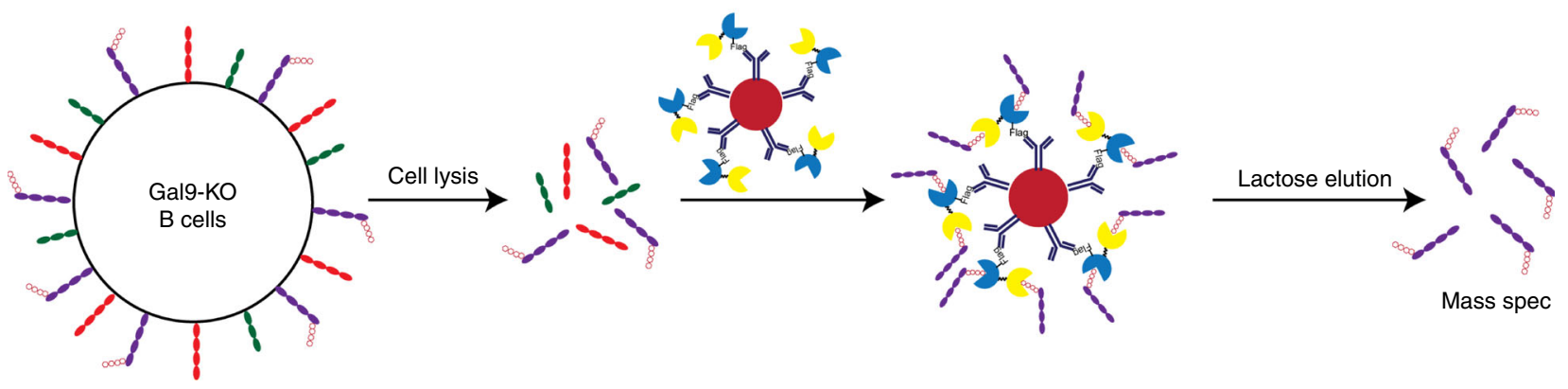

b

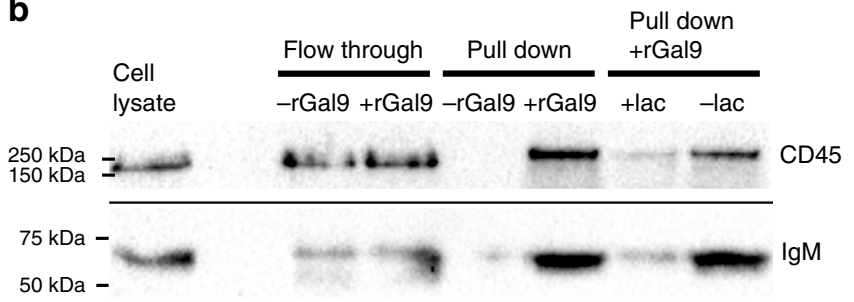

C

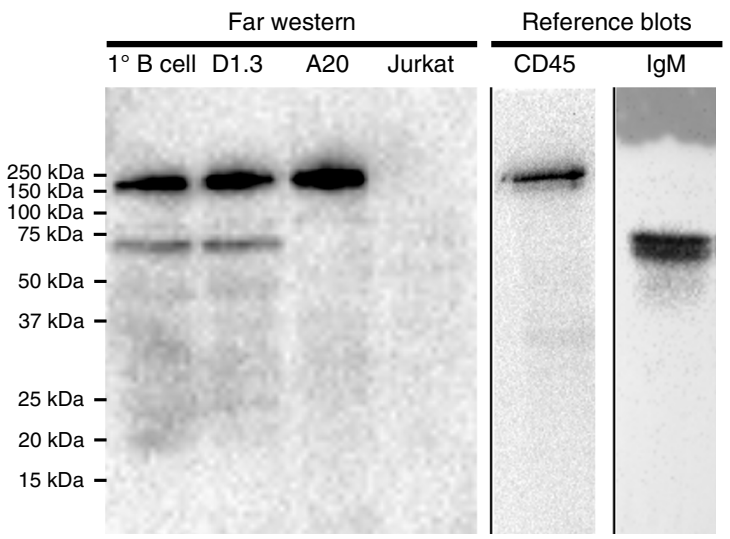

Fig. 4 Galectin-9 binds IgM-BCR and CD45. a Schematic diagram of pull-down assay using FLAG-tagged rGal9 coupled to magnetic beads. Galectin-9interacting ligands were eluted by treatment with lactose and then identified by mass spectrometry. $\mathbf{b}$ Lactose eluted proteins from beads coated ( $+\mathrm{rGal} 9$ ) or not ( $-r$ Gal9) with rGal9, or in the presence of lactose (+lac) or not ( - lac) were subjected to SDS-PAGE following by immunoblotting using antibodies specific to CD45 and IgM. c Cell lysates from primary murine B cells (lane 1), A20 B cells expressing Hel-specific lgM (D1.3; lane 2), A20 B cells (lane 3), and Jurkat T cells (lane 4) were lysed and subjected to far-western analysis using FLAG-tagged rGal9. Reference blots probed for CD45 and IgM are shown on the right. Data representative of three independent experiments

calcium upon addition of rGal9 (Supplementary Fig. 5). This finding raises the question of why treatment with rGal9, which clusters BCR, does not itself induce B-cell activation. Our data also identified CD45, the most abundant phosphatase on the Bcell surface, as a ligand of galectin-9 (Fig. 4). CD45 is a member of the protein tyrosine phosphatase family and plays an important role in both positive and negative regulation of B-cell activation through dephosphorylation of the activating and inhibitory sites of the Src family kinase $\operatorname{Lyn}^{40}$. This dual regulation of BCR signaling by CD45 is dependent on the localization of CD45, as CD45 constitutively dephosphorylates and inactivates Lyn within glycosphingolipid-enriched domains (GEMs), and BCR ligation induces sequestration of CD45 from GEMs ${ }^{41}$. We predicted that galectin-9 modifies the spatial organization of IgM and CD45 to attenuate signaling. To visualize the effect of galectin- 9 on organization of CD45 relative to IgM-BCR, we treated primary WT B cells with $1 \mu \mathrm{M}$ rGal9 and then fixed and immunostained using anti-CD45, anti-IgM, and anti-galectin-9 antibodies, and imaged by confocal microscopy. Interestingly, rGal9 often forms a cap at one side of the cell, consistent with the ability of galectins to form a lattice-like structure through galectin-glycoprotein interactions (Fig. 7a). We also observed that within the galectin-9 cap, the fluorescence intensity of CD45 and IgM is increased (Fig. 7a, b). To quantify this observation, we developed a protocol to define galectin-9-high regions (Gal9high) and galectin-9-low regions $\left(\mathrm{Gal} 9^{\text {low }}\right.$ ) (Fig. 7c) and found that the mean fluorescence intensity of both CD45 and IgM is significantly higher in Gal9high regions compared to Gal9 $9^{\text {low }}$ regions (Fig. $7 \mathrm{~d}$ ).

Reorganization of IgM and CD45 is not due to a global change in membrane structure, as the intensity of $\operatorname{IgD}$, an isotype of BCR that is expressed 10 -fold higher than $\operatorname{IgM}^{9}$, was not increased within the galectin-9 lattice (Supplementary Fig. 6a-c).

One of the best characterized inhibitory co-receptors expressed on B cells is CD22 2 . The extracellular domain of CD22 is also glycosylated and contains a sialic acid-binding domain, which binds a2,6-linked sialic acids and mediates homotypic interactions between CD22 molecules and heterotypic interactions with other glycosylated proteins, including CD45 and $\operatorname{IgM}^{43,44}$. Thus, we investigated if rGal9 also alters organization of CD22. We observed clusters of CD22 with higher fluorescence intensity inside the galectin-9 cap, which colocalized with IgM clusters (Fig. 7e, f). Consistent with this, the mean fluorescence intensity of CD22 is increased inside Gal9high regions (Fig. 7g). These findings suggest that rGal9 increases the density of CD22, coincident with the enrichment of $\operatorname{IgM}$, within the galectin-9 lattice. Again, to confirm the specificity of CD22 enrichment within the galectin-9 lattice, we examined localization of inhibitory receptor Fc $\gamma$ RIIb, upon treatment with rGal9, and found no enrichment within Gal9high regions (Supplementary Fig. 6d-f).

Our findings suggest that galectin- 9 enhances the interaction between IgM and CD22 and may provide a mechanistic basis for 
Table 1 Summary table of top hits of galectin-9-binding proteins identified by MS

\begin{tabular}{|c|c|c|c|c|c|c|c|c|c|}
\hline UniprotKB ID & Gene & $\log (e)$ & $\log (D)$ & $\%$ Measured & $\%$ Corrected & $\begin{array}{l}\text { Unique } \\
\text { peptides }\end{array}$ & $\begin{array}{l}\text { Total } \\
\text { peptides }\end{array}$ & $\mathbf{M r}$ & $\begin{array}{l}\text { Total peptides/ } \\
\text { molecular weight }\end{array}$ \\
\hline Q8VDN2 & Atpla1 & -165.4 & 5.7 & 17 & 31 & 12 & 29 & 112.9 & 0.256864482 \\
\hline Q8C129 & Lnpep & -119.5 & 5.58 & 11 & 23 & 10 & 21 & 117.2 & 0.179180887 \\
\hline P06800 & Ptprc & -114.7 & 5.72 & 9.2 & 17 & 11 & 25 & 144.5 & 0.173010381 \\
\hline P24668 & M6pr & -75.3 & 5.8 & 21 & 36 & 5 & 16 & 31.2 & 0.512820513 \\
\hline Q03265 & Atp5a1 & -70.2 & 5.33 & 18 & 24 & 7 & 12 & 59.7 & 0.201005025 \\
\hline P11835 & Itgb2 & -63.2 & 4.98 & 6.8 & 9 & 4 & 7 & 84.8 & 0.08254717 \\
\hline P19437 & Ms4a1 & -60.9 & 5.71 & 22 & 60 & 5 & 25 & 31.9 & 0.78369906 \\
\hline P10852 & Slc3a2 & -59.2 & 5.34 & 13 & 21 & 5 & 12 & 62.2 & 0.192926045 \\
\hline Q8BG07 & Pld4 & -52.3 & 5.3 & 16 & 32 & 5 & 11 & 56.1 & 0.196078431 \\
\hline P01872 & lghm & -39.3 & 5.3 & 8.6 & 16 & 3 & 9 & 50 & 0.18 \\
\hline Q62192 & Cd180 & -36.1 & 5.19 & 6.7 & 14 & 4 & 9 & 74.3 & 0.121130552 \\
\hline P51881 & Slc25a5 & -32.7 & 5.77 & 7 & 10 & 3 & 8 & 32.9 & 0.243161094 \\
\hline Q61735 & $C d 47$ & -25.6 & 5.39 & 7.7 & 26 & 2 & 11 & 35.3 & 0.311614731 \\
\hline P15530 & $C d 79 b$ & -18.8 & 5.11 & 7.6 & 22 & 2 & 7 & 32.1 & 0.218068536 \\
\hline O35424 & $\mathrm{H} 2-\mathrm{Ob}$ & -15.4 & 5.18 & 6.6 & 10 & 2 & 6 & 30.4 & 0.197368421 \\
\hline
\end{tabular}

Relevant statistics from one replicate experiment. Top hits here identified using the following criteria: $\log ($ e) score $\leq-10$; total peptides $\geq 5$; unique peptides $\geq 2$ in at least two independent experiments. Hits observed in "no bait" controls were removed. Data acquired with input of $2 \mathrm{e} 7$ cells and $200 \mathrm{mM}$ lactose elution

the inhibitory effect of galectin-9 on B-cell activation. The intracellular domain of CD22 contains three immunoreceptor tyrosine-based inhibitory motifs that are phosphorylated upon BCR ligation ${ }^{43}$ and recruit other phosphatases such as Src homology region 2 domain-containing phosphatase-1 (SHP-1), which dephosphorylates Syk, PLC $\gamma 2$, and CD19 to dampen BCR signaling ${ }^{45}$. Given our finding of reduced phosphorylation of CD19 in BCR stimulated cells pre-treated with rGal9 (Fig. 3d), we examined if CD19 is also localized within the galectin-9 lattice, and found that CD19 is indeed enriched within Gal9high regions (Fig. 7h-j). These findings suggest that galectin-9 reorganizes IgM-BCR and its co-receptor CD19 together with inhibitory coreceptors CD45 and CD22 to suppress BCR signaling.

GEMs (or lipid rafts) play an important role in initiation of BCR signaling, and the localization of proteins within these domains have important consequences for activation of signaling ${ }^{46}$. We found that when B cells are treated with rGal9, galectin- 9 often forms a cap on one side of the cell, reminiscent of the polarization of lipid rafts upon lymphocyte activation ${ }^{47,48}$. Thus, we asked whether galectin-9 alters the distribution of lipid rafts and association of CD45 and CD22 with these domains. We treated primary B cells with rGal9 and then labeled lipid rafts with fluorescently conjugated cholera toxin $\beta$-subunit (CT-B) and immunostained for galectin-9, CD45, and CD22. Treatment with rGal9 increased the intensity of CT-B within galectin-9 regions and this region was enriched for CD45 and CD22 (Fig. 8a, b, e, f). We generated masks defining CT-B-high (lipid raft high, LR high) and CT-B-low (lipid raft-low regions, $\mathrm{LR}^{\mathrm{low}}$ ) regions and then examined the intensity of CD45 and CD22 within these regions (Fig. 8c). The mean intensity of both CD45 and CD22 is increased within lipid raft-rich regions upon treatment with rGal9 (Fig. 8d, g). Together with our previous findings, these data suggest that treatment with $\mathrm{rGal} 9$ induces coalescence of lipid raft domains containing CD45 and CD22 to suppress CD19 and BCR activation.

Galectin-9 mediates association of IgM and CD22. We used rGal9 as a tool to understand the molecular mechanism for enhanced B-cell activation in galectin-9-deficient B cells. These studies revealed that $\mathrm{rGal} 9$ alters the organization of CD45 and CD22 with respect to IgM-BCR (Fig. 7) and this correlates with suppressed BCR signaling (Fig. 3). To investigate if lack of galectin- 9 altered association of IgM-BCR with these proteins in the steady state, we employed dual-color dSTORM to examine colocalization of CD45 and CD22 with IgM-BCR in WT and Gal9-KO B cells. We validated our dual-color dSTORM imaging and analysis parameters by labeling IgM with both anti-IgM coupled to Alexa 488 and Alexa 647 (Supplementary Fig. 7). We labeled primary naive B cells from WT and Gal9-KO mice with Alexa 488-labeled IgM-specific Fab fragment and Alexa 647labeled anti-CD45 (B220) or anti-CD22 and settled them on antiMHC II-coated coverslips. Dual-color dSTORM revealed that CD45 is localized in nanoscale clusters, which minimally overlap with IgM clusters in WT B cells, with no obvious difference compared to Gal9-KO cells (Fig. 9a). We also found no difference in the Hopkins index, $H$ function curve, mean cluster diameter, and mean cluster area of CD45 in WT and Gal9-KO B cells (Fig. 9b-e). To examine colocalization of IgM and CD45 we performed coordinate-based colocalization (CBC) analysis $^{49}$, which ranges from -1 (perfectly segregated) to +1 (perfectly colocalized). CBC analysis of WT cells revealed a distribution histogram with two peaks, one approaching -1 and one approaching +1 , indicating some degree of colocalization between IgM and CD45 in the resting state; however, the median $\mathrm{CBC}$ value for IgM and CD45 was 0.018 indicating that across the population of molecules the distribution is largely uncorrelated (Fig. 9f). We observed a similar distribution of correlation coefficients and nearest-neighbor distance in Gal9-KO B cells (Fig. 9f, g), suggesting that galectin-9 does not influence the association of IgM and CD45 to a significant degree in the steady state.

In contrast to CD45, dual-color dSTORM of IgM and CD22 showed a larger degree of colocalization based on visual inspection of dSTORM images (Fig. 9h). Consistent with previous reports ${ }^{50} \mathrm{CD} 22$ is highly clustered on the surface of resting primary naive $B$ cells. We noted a small but statistically insignificant difference in both the mean Hopkin's index and the $H$ function curve, with CD22 appearing slightly less clustered in Gal9-KO B cells compared to WT cells (Fig. 9i, j). However, we noted no difference in mean cluster diameter or cluster area of CD22 between WT and Gal9-KO B cells (Fig. 9k, 1). CBC analysis of colocalization of $\operatorname{IgM}$ and CD22 revealed that the frequency distribution was right shifted in WT B cells, indicating that CD22 is more colocalized with $\operatorname{IgM}$ than CD45 in the steady state (Fig. 9m). Indeed, the median CBC value in WT cells was 0.22 . In contrast, the median $\mathrm{CBC}$ value in Gal9-KO B cells was 0.12 , and the nearest-neighbor analysis revealed that the mean distance between IgM and CD22 molecules was increased (Fig. 9m, n). 
a
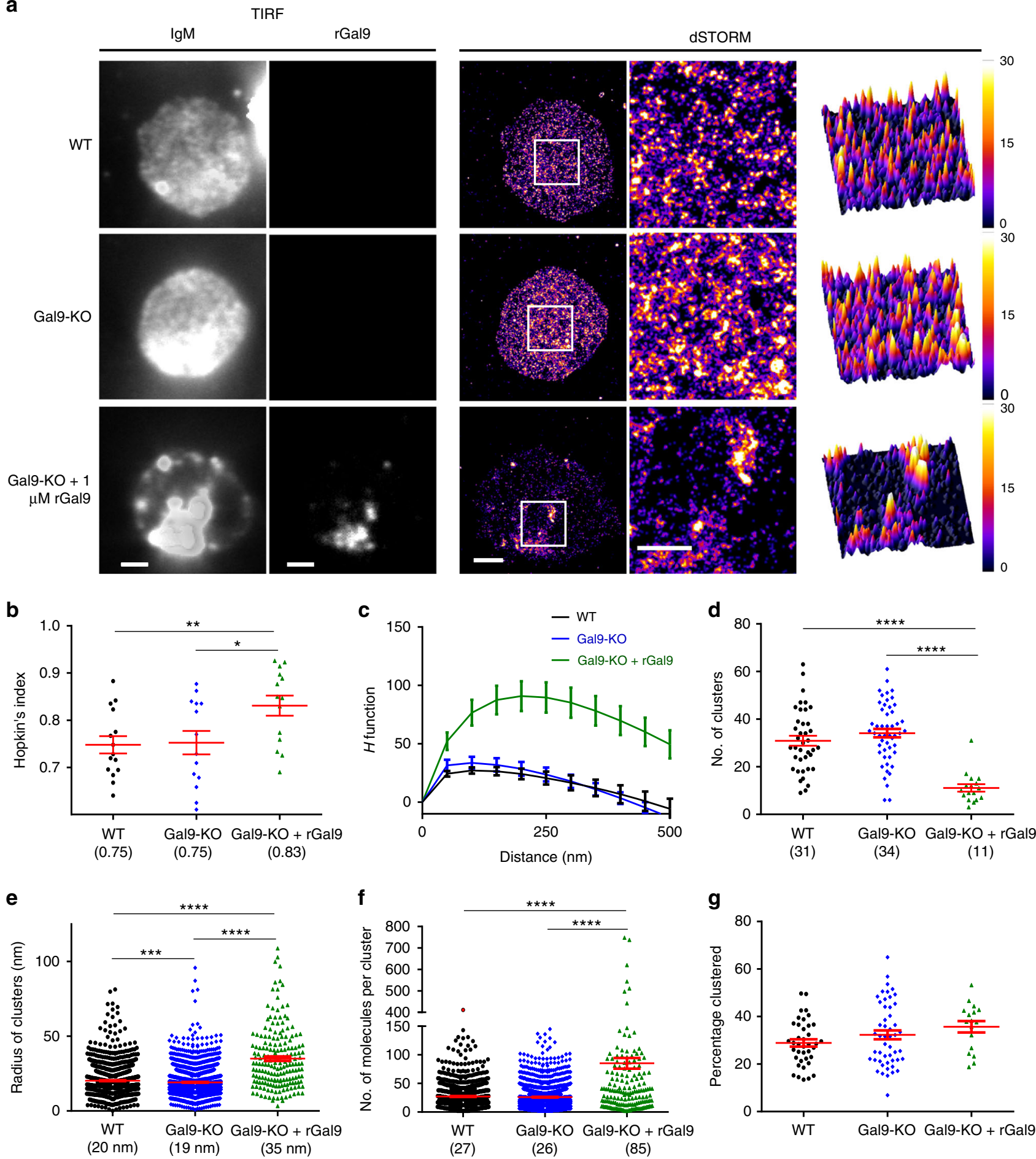

Fig. 5 Galectin-9 alters IgM-BCR nanoclusters. a TIRFM image of surface IgM and fluorescently labeled rGal9 before bleaching for image acquisition (two left panels respectively). dSTORM images reconstructed from single-molecule localization processed by Thunderstorm software mapped to a fire color scale as indicated; the magnified region $(3 \times 3 \mu \mathrm{m})$ from ROI (white box) is shown as 2D image (middle) and 3D surface plot (right) in the order of WT (top), Gal9-KO (middle), and Gal9-KO $1 \mu \mathrm{M}$ rGal9 (bottom). Scale bar represents $2 \mu \mathrm{m}$. $\mathbf{b}$ Quantification of the distribution of IgM by $H$ function and c Hopkins index of localizations inside ROls. $\mathbf{d}-\mathbf{g}$ Reconstructed images were analyzed by a model-based Bayesian approach to identify nanoclusters and their physical properties. $\mathbf{d}$ Number of clusters (one point per ROI). e Cluster radii (one point per cluster). $\mathbf{f}$ Number of molecules (one point per cluster). g Percentage of localization in clusters (one point per ROI). Each category contains at least 15 ROls from three independent experiments (at least four cells per experiment). Statistical analysis was performed using Kruskal-Wallis test with Dunn's multiple comparison test (d, e, f) and one-way ANOVA with Tukey's multiple comparison test $(\mathbf{b}, \mathbf{g})$. Red bars indicate mean $\pm \mathrm{SEM} ;{ }^{\star} p<0.05,{ }^{\star \star} p<0.01,{ }^{\star \star \star} p<0.001,{ }^{\star \star \star \star} p<0.0001$ 

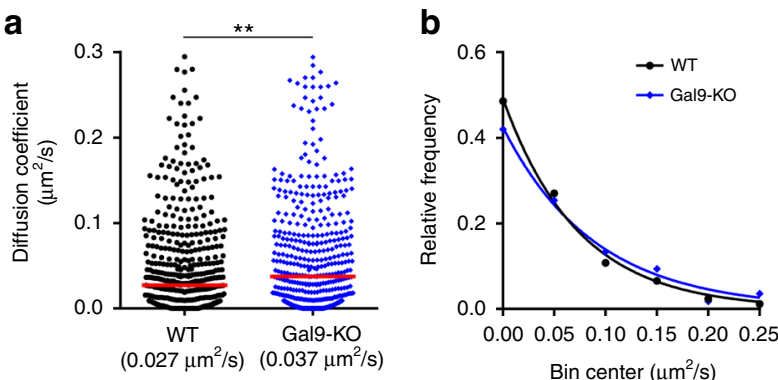

C
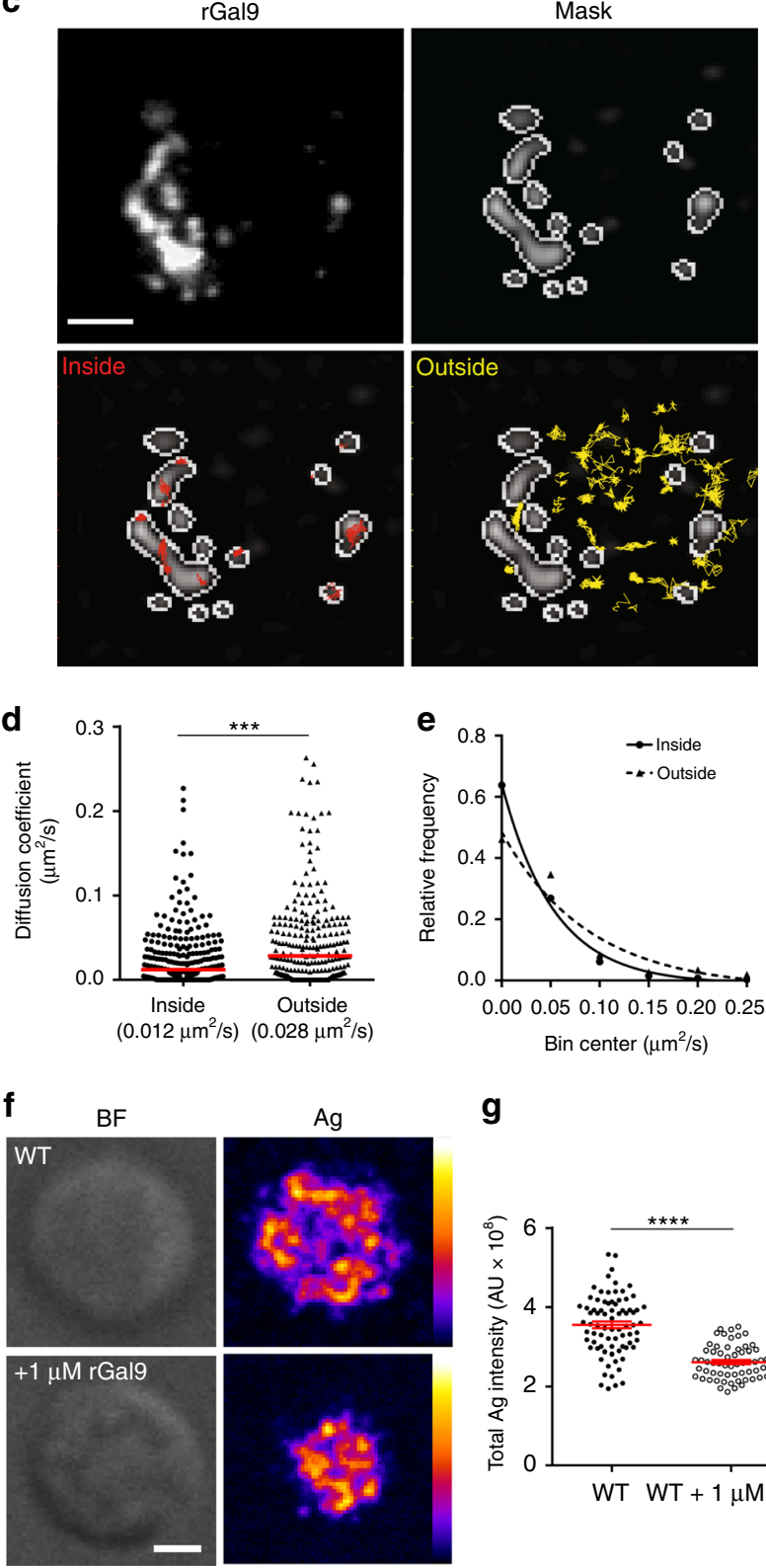

g

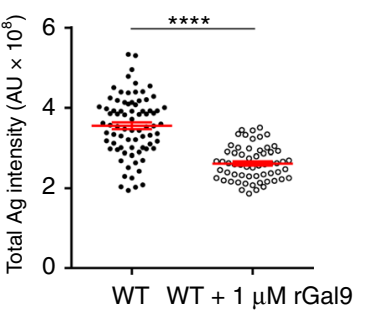

These findings suggest that galectin- 9 mediates close association between IgM and CD22, at least to some extent, in the steady state.

\section{Discussion}

Here we identify galectin-9 as a negative regulator of B-cell activation. We found that BCR microcluster formation and signaling are enhanced in galectin-9-deficient B cells. Importantly,
Fig. 6 Galectin-9 immobilizes IgM-BCR and attenuates BCR microclustering. a Diffusion coefficients and $\mathbf{b}$ frequency distribution histogram of single-particle tracking of IgM in WT (black circle) or Gal9-KO (blue diamond) primary $B$ cells with the median indicated in red. Five hundred representative diffusion coefficients from a total of at least 1500 tracks from three independent experiments. c Representative TIRF image of fluorescently labeled rGal9 on primary B cell (left) and mask (right) created to differentiate tracks inside Gal9 regions (lower left, red lines) and tracks outside Gal9 regions (lower right, yellow lines). d Diffusion coefficients and e frequency distribution inside Gal9 regions (black circle) and outside Gal9 regions (black triangle) with the median indicated in red. In all, 250 representative diffusion coefficients from a total of at least 900 tracks from three independent experiments. f Representative TIRF microscopy images of WT cell (top) and WT cells treated with $1 \mu \mathrm{M} \mathrm{rGal9}$ (bottom) on artificial planar lipid bilayers containing anti-kappa mapped to an 8-bit fire color scale (ImageJ). $\mathbf{g}$ Quantification of the total antigen intensity at the cellbilayer interface in WT (black circles) and WT treated with rGal9 (open circles), with the mean \pm SEM indicated by the red bar. Scale bar represents $2 \mu \mathrm{m}$. Statistical significance assessed by Mann-Whitney; ${ }^{\star \star \star \star} p<$ $0.0001,{ }^{\star \star \star} p<0.001,{ }^{\star \star} p<0.01$

we show that the ligands of galectin- 9 at the cell surface are not saturated in freshly isolated naive B cells and treatment with rGal9 nearly abolishes BCR signaling. Using a pull-down assay and mass spectrometry we identified IgM and CD45 as ligands of galectin-9 in primary B cells. We further show that galectin-9 plays a role in regulating the spatial organization and dynamics of IgM-BCR and its localization with regulatory surface proteins. We propose that galectin- 9 organizes IgM-BCR into larger clusters that restrict the mobility of IgM and relocalizes inhibitory molecules, including CD45 and CD22 to directly inhibit BCR signaling (Fig. 10).

The spatial organization of cell surface proteins in constitutive nanoscale clusters has emerged as a general principle of cell surface proteins ${ }^{51}$. However, the molecular mechanism regulating the size, composition, and stability of these assemblies is an open question. The formation and stability of IgM-BCR nanoclusters is not dependent on the actin cortex, as depolymerization of actin does not alter the size or density of IgM-BCR nanoclusters9. Thus, we hypothesized that the galectin- 9 lattice may play a role in the nanoscale organization of IgM-BCR. In contrast, superresolution SSTORM revealed no difference in the size or density of IgM-BCR nanoclusters in galectin-9-deficient B cells. This is consistent with a recent study demonstrating that glycan-based interactions do not mediate the nanoclustering of dendritic cellspecific intercellular adhesion molecule-3-grabbing nonintegrin $^{52}$. It may be that formation of BCR nanoclusters (or oligomers) is largely dependent on the direct interaction of BCR components, as suggested by Reth and colleagues ${ }^{16,53}$. However, we do find that addition of rGal9 induces significant changes in IgM-BCR organization. Inside the galectin-9 lattice, the number of IgM-BCR nanoclusters decreased while the radius of clusters, and the number of molecules in each cluster, increased. Thus, we propose that galectin-9 provides a second layer of organization to IgM-BCR by merging pre-existing nanoclusters.

But how does this observation square with our finding that rGal9 suppresses BCR signaling? Indeed, these observations are somewhat counter-intuitive given that $\mathrm{BCR}$ stimulation induces the coalescence of BCR nanoclusters to form larger-scale signaling microclusters ${ }^{9}$. So, how does the larger cluster of IgM induced by galectin- 9 not activate B cells, but instead inhibit BCR signaling? We propose two potential mechanisms for galectin-9mediated control of BCR signaling. First, we propose that galectin-9 induced merging of pre-existing nanoclusters decreases 
a
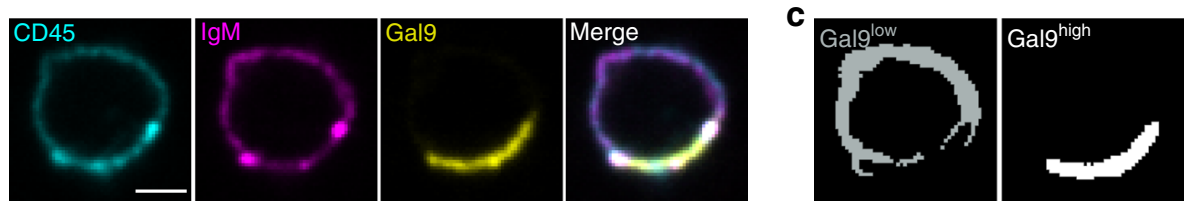

b

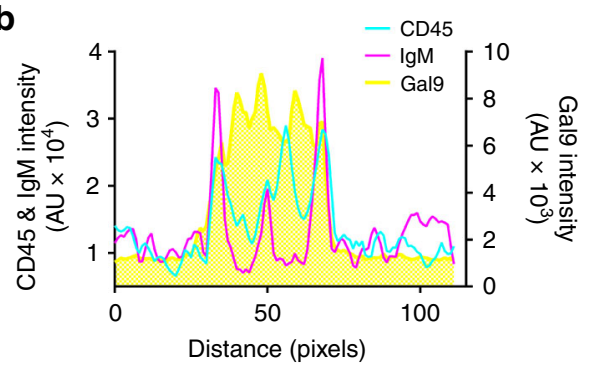

d
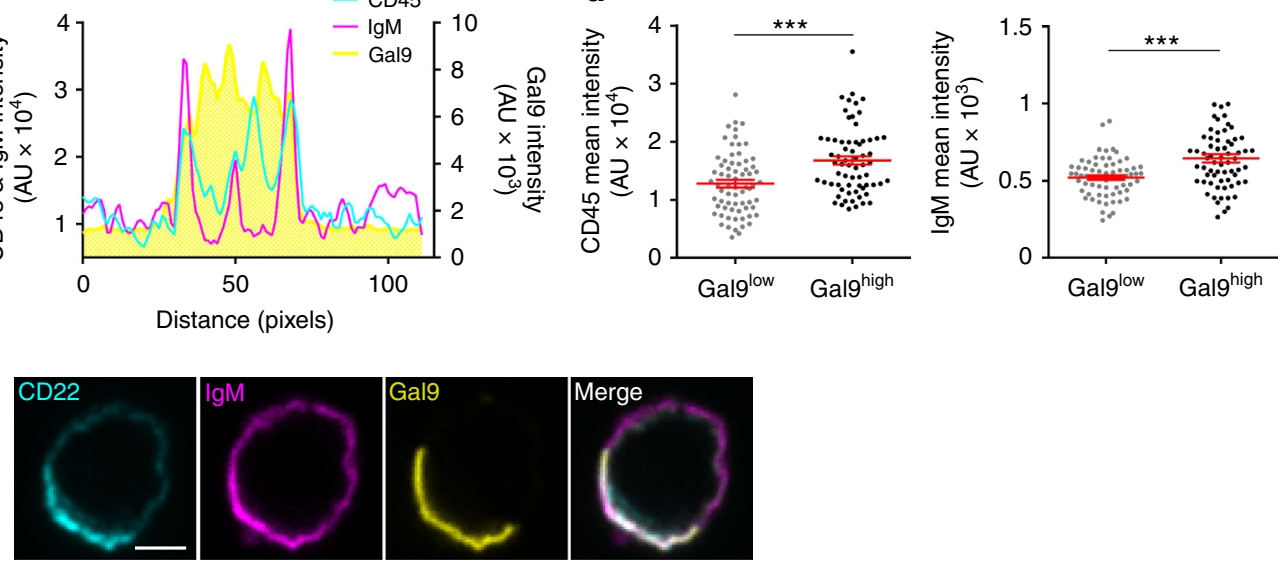

f

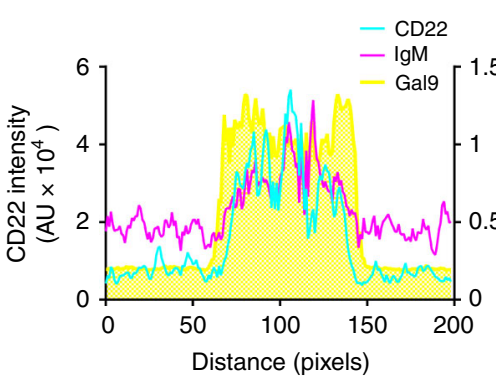

g

h
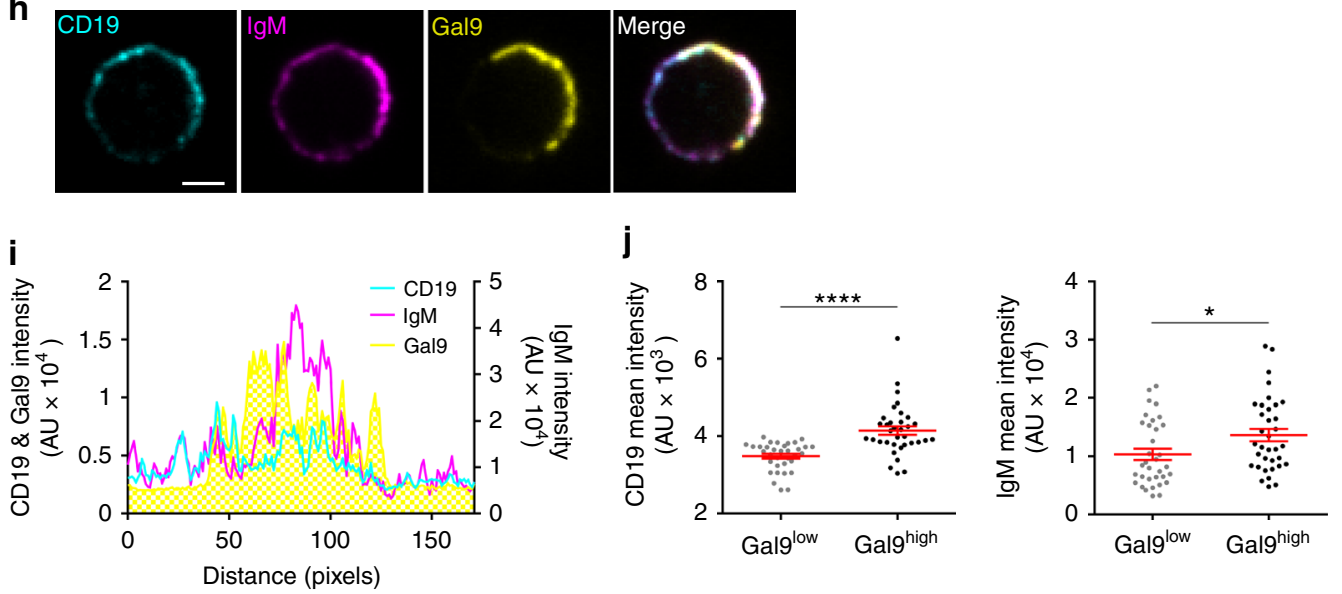

Fig. 7 The galectin-9 lattice increases the molecular density of IgM-BCR and co-receptors. a Representative confocal images of primary WT B cells treated with $1 \mu \mathrm{M}$ rGal9 and immuostained for CD45 (cyan), IgM (magenta), and galectin-9 (Gal9; yellow). b Fluorescence intensity profile of CD45, IgM, and Gal9 along the cell membrane. c Representative example of masking output of algorithm to detect regions of high galectin-9 (Galghigh) and low galectin-9 (Galglow). d Mean fluorescence intensity of CD45 (left) and lgM (right) in Galghigh and Galglow regions. e Representative confocal images of WT B cells treated with $1 \mu \mathrm{M}$ rGal9 and immunostained for CD22 (cyan), IgM (magenta), and Gal9 (yellow). f Fluorescence intensity profile of CD22, IgM, and Gal9 along the cell membrane. $\mathbf{g}$ Mean fluorescence intensity of CD22 (left) and lgM (right) in Galghigh and Galglow regions. $\mathbf{h}$ Representative confocal images of WT B cells treated with $1 \mu \mathrm{M}$ rGal9 and immunostained for CD19, IgM, and Gal9. i Fluorescence intensity profile of CD19, IgM, and Gal9 along the cell membrane. j Mean fluorescence intensity of CD19 (left) and IgM (right) in Gal9low and Galghigh regions. Data representative of at least three independent experiments. Each dot represents 1 cell, at least 30 cells measured per condition per experiment. Mean \pm SEM indicated by the red bar. Statistical significance assessed by Mann-Whitney; ${ }^{\star \star \star \star} p<0.0001{ }^{\star \star \star} p<0.001,{ }^{\star} p<0.05$. Scale bar $2 \mu \mathrm{m}$ 

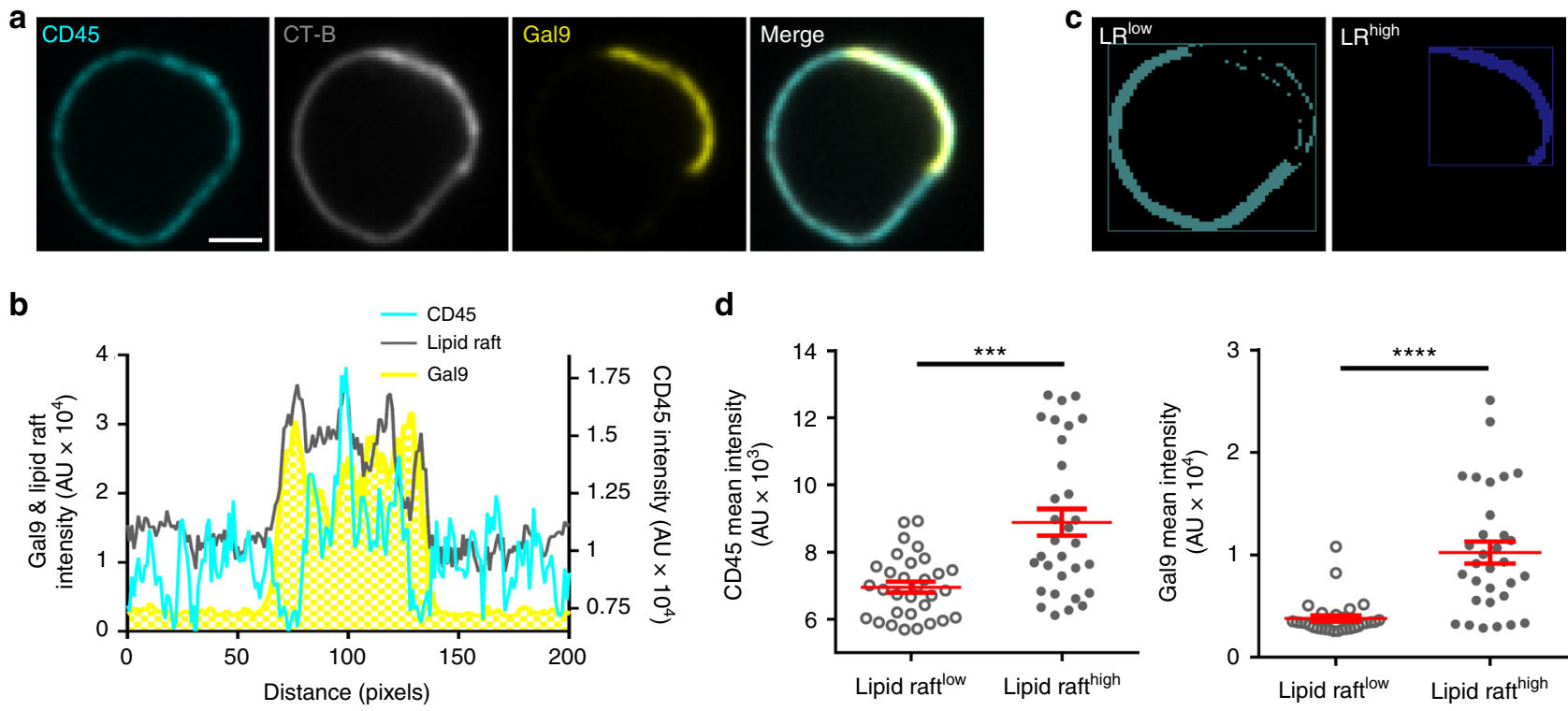

d
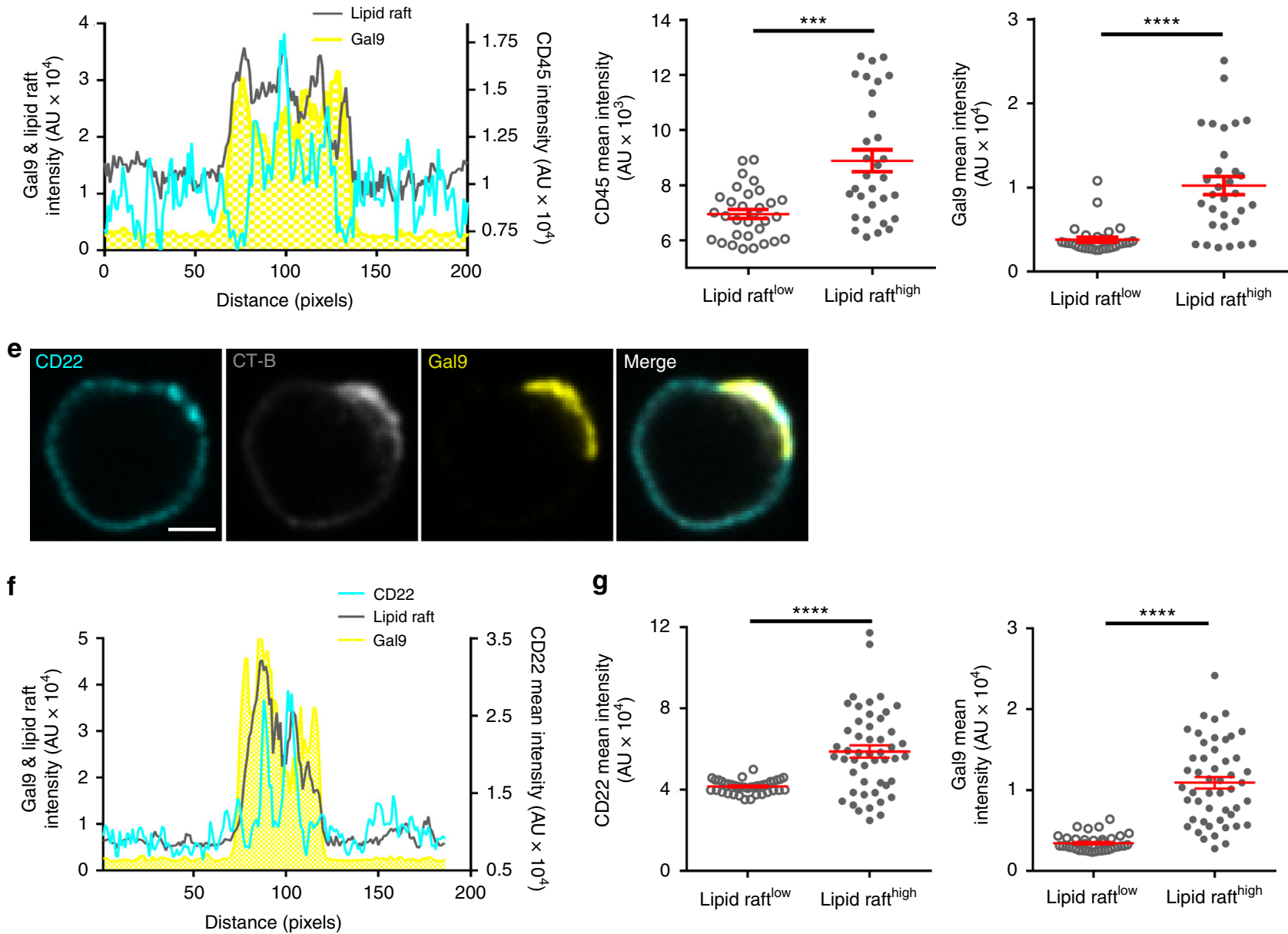

Fig. 8 rGal9 induces coalescence of lipid raft domains containing CD22 and CD45. a Representative confocal images of primary WT B cells treated with 1 $\mu \mathrm{M}$ rGal9 and immunostained for CD45 (cyan) and galectin-9 (Gal9; yellow), and fluorescent cholera toxin (CT-B; gray) to label lipid rafts. b Fluorescence intensity profile of CD45, CT-B, and Gal9 along the cell membrane. c Representative example of masking output of algorithm to detect regions of high CT-B (lipid raft high; $L R^{\text {high }}$ ) and low CT-B (LR low). d Mean fluorescence intensity of CD45 (left) and Gal9 (right) in $L R^{\text {low }}$ and $L R^{\text {high }}$ regions. e Representative confocal images of WT B cells treated with $1 \mu \mathrm{M}$ rGal9 and immunostained for CD22 (cyan), Gal9 (yellow), and fluorescent CT-B (gray). f Fluorescence intensity profile of CD22, CT-B, and Gal9 along the cell membrane. $\mathbf{g}$ Mean fluorescence intensity of CD22 (left) and Gal9 (right) in LR low and LR high regions. Data are representative of at least three independent experiments. Each dot represents 1 cell, at least 30 cells measured per condition per experiment. Mean \pm SEM indicated by the red bar. Statistical significance assessed by Mann-Whitney; ${ }^{\star \star \star \star} p<0.0001$, ${ }^{\star \star \star} p<0.001$. Scale bar $2 \mu \mathrm{m}$

the lateral diffusion of IgM-BCR and consequently suppresses BCR signaling. The mobility of IgM-BCR on the cell membrane can be described as Brownian motion restricted by the underlying actin cytoskeleton $^{12,54}$. The theory of Brownian motion postulates that the larger the particle the slower the movement of the particle $^{55}$. Thus, we would predict that larger clusters of IgM-BCR inside the galectin-9 lattice have a lower diffusion coefficient compared to smaller clusters outside the galectin-9 lattice, consistent with our data. This interpretation is supported by the finding that in CD45-deficient B cells, homotypic clustering of CD22 is enhanced, which correlates with decreased lateral diffusion $^{50}$. In addition, IgD-BCR is more densely clustered than IgM-BCR ${ }^{9}$ and this correlates with a 10 -fold lower diffusion coefficient compared to IgM-BCR ${ }^{12}$. Thus, we propose that galectin-9 restricts IgM-BCR mobility by organizing IgM-BCR into larger clusters. The mobility of BCR on the cell surface is correlated with BCR signaling; simply depolymerizing actin increases BCR mobility and triggers spontaneous signaling ${ }^{12}$. Consistent with this, treating B cells with lipopolysaccharide increases actin severing and BCR mobility, which lowers the threshold for B-cell activation ${ }^{54}$. Increased diffusion of the BCR (or BCR nanoclusters) may be important for BCRs to join newly forming microclusters. In agreement with this, treatment with rGal9 decreased BCR microcluster formation upon stimulation by membrane-bound antigen. Conversely, increased BCR diffusion would be associated with more microclusters, in line with our 
a
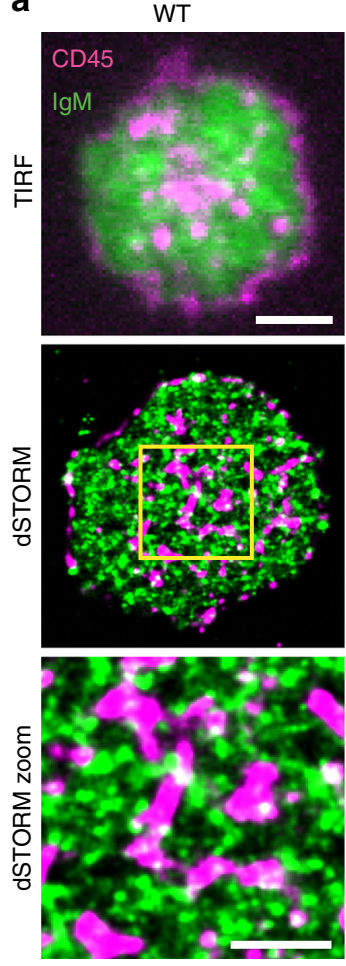

h
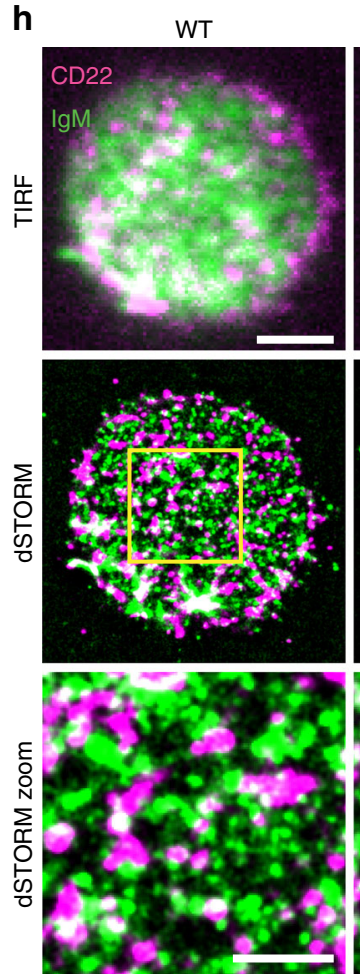

Gal9-KO
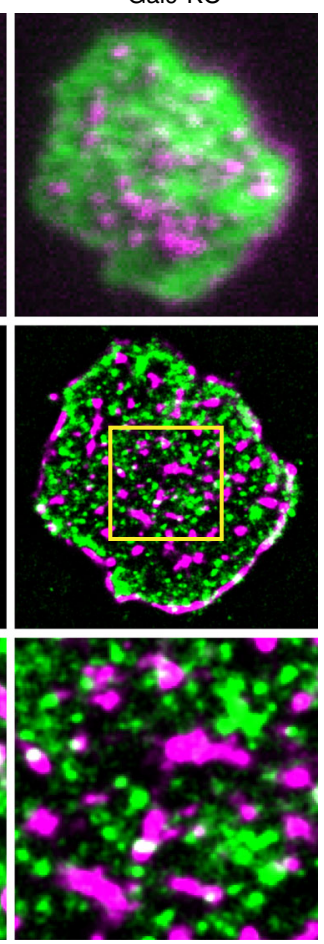

Gal9-KO
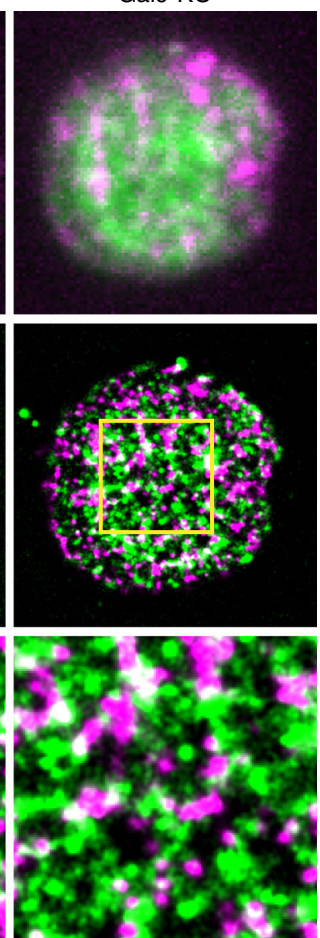

b
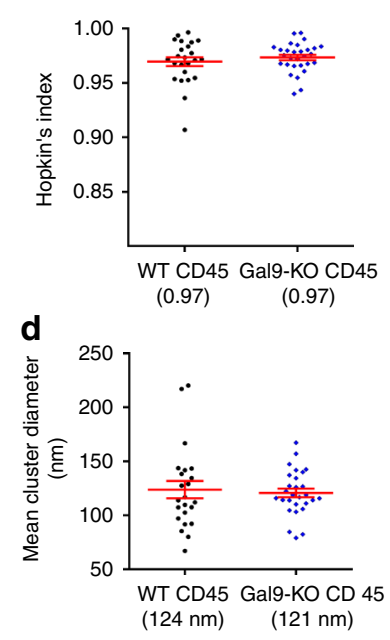

f

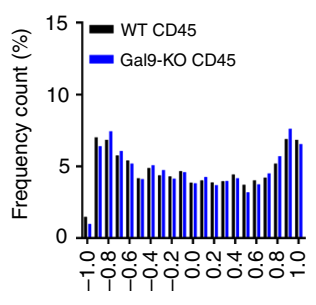

Bin center

i
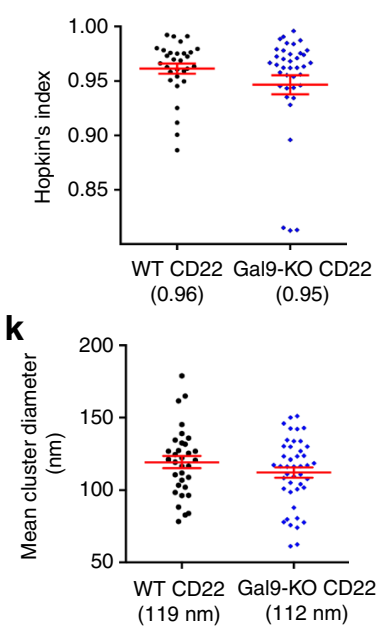

m

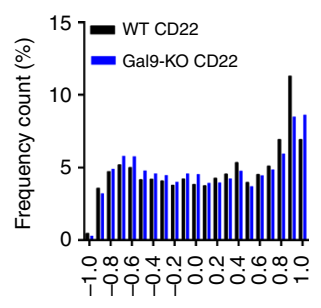

Bin center
C

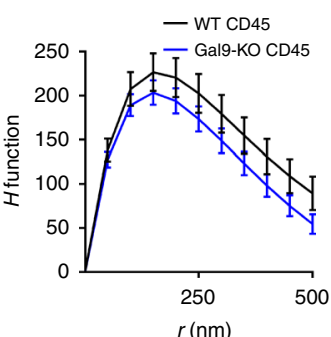

e

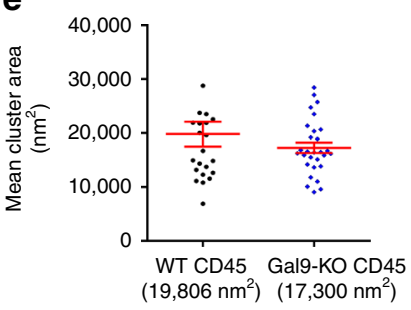

g

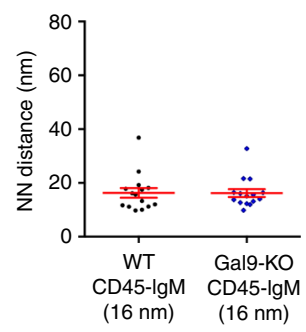

j
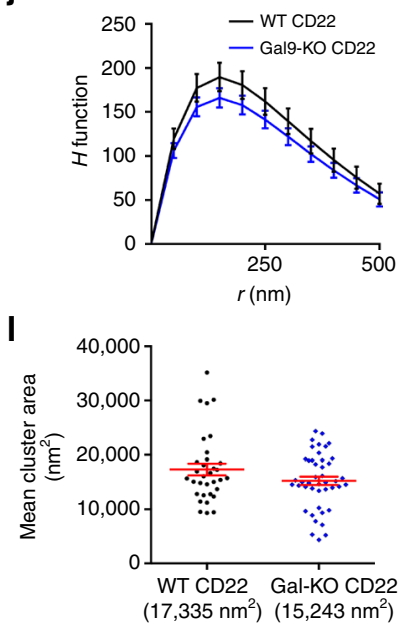

n

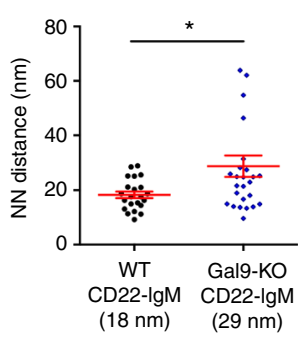

observation of increased BCR mobility in galectin-9-deficient B cells and enhanced B-cell activation.

Additionally, we propose that galectin-9 induced merging of BCR nanoclusters regulates the interaction between IgM and the co-receptors CD45 and CD22 to suppress BCR signaling. Our finding of CD45 enrichment in the galectin-9 lattice is consistent with our identification of CD45 as a ligand of galectin-9. The
$\mathrm{N}$-terminal of CD45 has multiple $\mathrm{N}$-glycosylation sites, providing potential binding sites for galectin family members. Indeed, in $\mathrm{T}$ cells, CD45 was reported to bind to galectin-1 ${ }^{56-58}$ and galectin- $9^{33}$. In addition, galectin-3 has been reported to bind to CD45 on diffuse large B-cell lymphoma ${ }^{59}$. These findings suggest that CD45 may be a promiscuous ligand of the galectin family, although differential glycosylation of CD45 in different immune 
Fig. 9 Galectin-9 increases colocalization between CD22 and IgM in primary B cells. a Representative merged TIRF (top), dSTORM (middle), and dSTORM zoom (bottom) images showing surface CD45 (magenta) and IgM-BCR (green) on primary wild-type (WT) (left) and galectin-9 knockout (Gal9-KO) (right) B cells. dSTORM ROI $(3 \times 3 \mu \mathrm{m})$ is outlined in yellow (middle) and magnified in dSTORM zoom (bottom). b-e Quantification of at least 20 ROls from WT and Gal9-KO B cells pooled from three independent experiments. b Hopkin's index showing randomness of CD45 organization (one point per ROI). $\mathbf{c} H$ function derived from Riplely's $K$ showing degree of CD45 clustering. d Mean diameter of CD45 clusters (one point per ROI). e Mean area of CD45 clusters (one point per ROI). $\mathbf{f}, \mathbf{g}$ Quantification of at least 15 ROls from WT and Gal9-KO B cells pooled from three independent experiments. $\mathbf{f}$ Coordinate-based colocalization (CBC) histograms of the single-molecule distributions of colocalizations between CD45 and IgM. $\mathbf{g}$ Nearest-neighbor distance (NND) analysis of the data shown in $\mathbf{f}$. Symbol represents the median NND of all paired single-molecule localizations from one ROI. h Representative merged TIRF and dSTORM images showing surface CD22 (magenta) and IgM-BCR (green) on primary WT (left) and Gal9-KO (right) B cells. i-I Quantification of at least 30 ROIs from WT and Gal9-KO B cells pooled from three independent experiments. i Mean Hopkin's index showing randomness of CD22 organization (one point per ROI). $\mathbf{j} H$ function showing degree of CD22 clustering. $\mathbf{k}$ Mean diameter of CD22 clusters (one point per ROI). I Mean area of CD22 clusters (one point per ROI). $\mathbf{m}, \mathbf{n}$ Quantification of at least 20 ROIs from WT and Gal9-KO B cells pooled from three independent experiments. $\mathbf{m}$ CBC histograms of the single-molecule distributions of colocalizations between CD22 and IgM. $\mathbf{n}$ NND analysis of the data shown in $\mathbf{m}$. Colocalization between channels shown in white. Scale bars represent 2 and $1 \mu \mathrm{m}$ (zoom). Mean \pm SEM indicated by the red bar. Statistical significance assessed by Mann-Whitney, ${ }^{*} p<0.05$

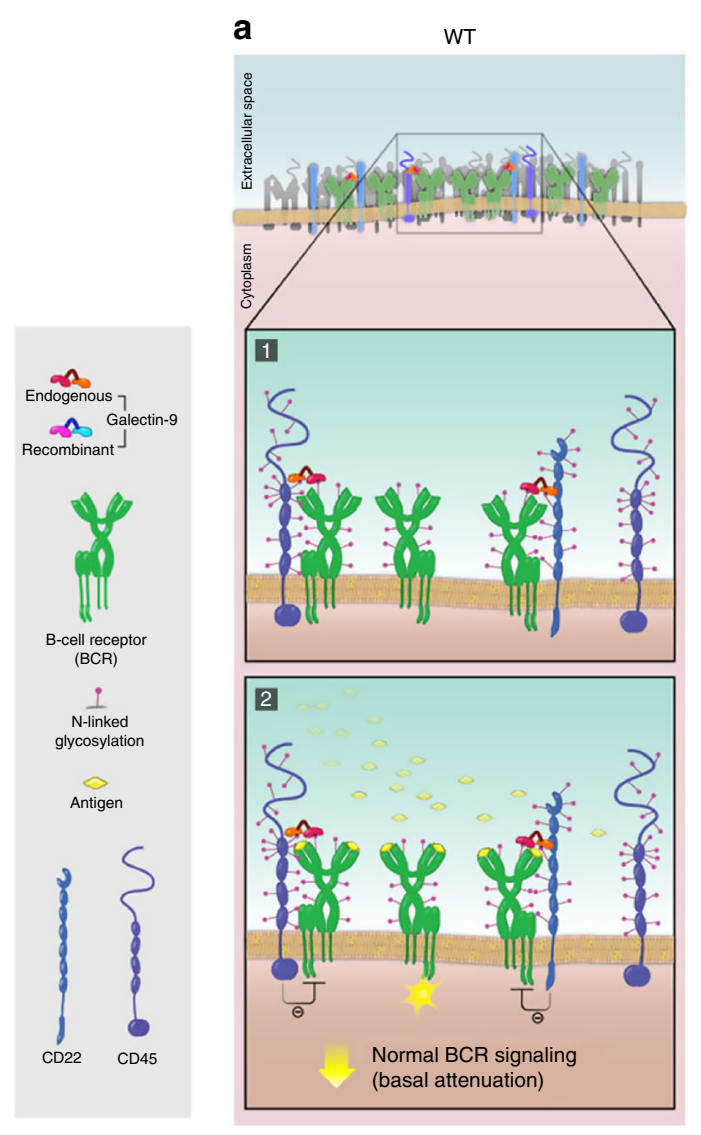

b
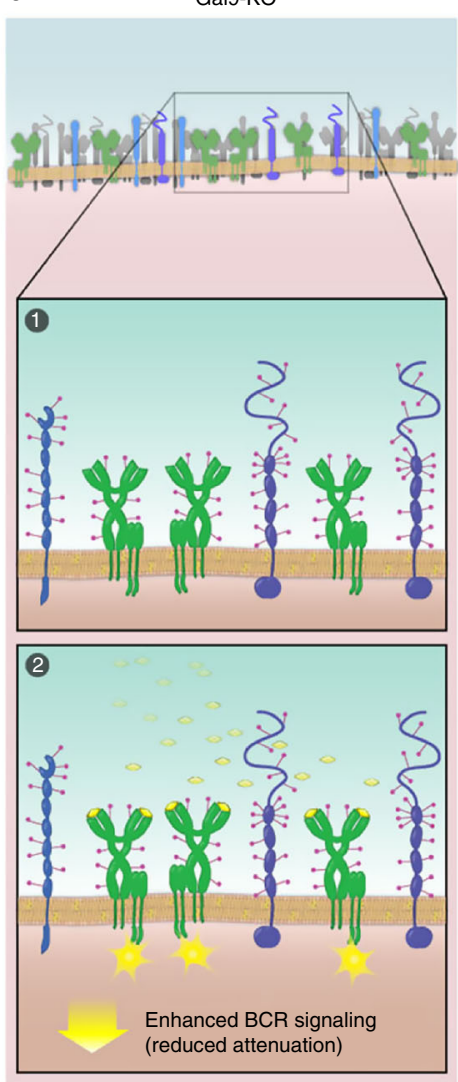

C
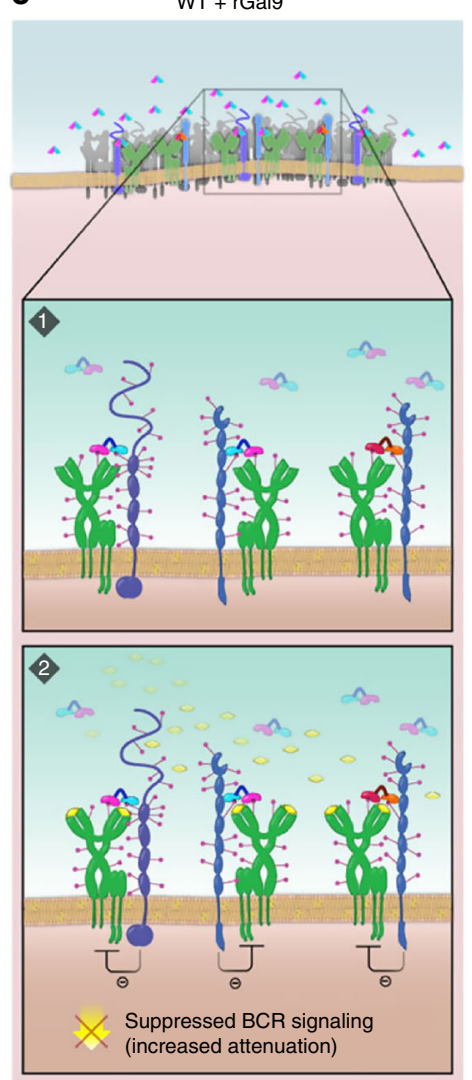

Fig. 10 Schematic model of galectin-9 regulation of B-cell activation. a In resting primary naive WT B cells, galectin-9 facilitates interactions between BCRs and either the inhibitory proteins CD45 or CD22 through binding to $\mathrm{N}$-linked glycans, providing a basal attenuation of B-cell signaling upon antigen stimulation. $\mathbf{b}$ BCR signaling is enhanced in Gal9-KO B cells due to loss of association of BCR with inhibitory co-receptors. c Treatment of WT B cells with rGal9 induces the association of IgM-BCR with CD45 and CD22 to suppress B-cell signaling

cell subsets may provide specificity for distinct galectin binding. Nonetheless, the enrichment of CD45 together with IgM inside the galectin-9 lattice is consistent with the inhibitory effect of galectin-9 on BCR signaling. Previous studies suggest that BCR activation is accompanied by the segregation of BCR and CD45 in order to promote Ig $\alpha-\operatorname{Ig} \beta$ phosphorylation ${ }^{41,60}$, consistent with the finding that CD45 is excluded from BCR microclusters ${ }^{2}$. Our finding that addition of rGal9 induces co-patching of CD45 and IgM and concomitant abrogation of BCR signaling is consistent with this model.
We also observed the enrichment of CD22 inside the galectin-9 lattice. CD22 is a known negative regulator of BCR signaling, which recruits the phosphatase SHP- $1^{45}$, which dephosphorylates CD1961 terminating the amplification of BCR signaling. In addition, SHP-1 dephosphorylates PLC $\gamma 2$ and attenuates MAPK activation $^{42}$. Thus, the localization of CD22 together with IgMBCR upon rGal9 treatment is consistent with our observation of reduced phosphorylation of CD19 and ERK1/2 and likely contributes to the inhibitory effect of galectin-9 on B-cell activation. Moreover, in resting naive $\mathrm{B}$ cells, dual-color dSTORM 
demonstrated that the colocalization of IgM and CD22 is reduced in galectin-9-deficient $\mathrm{B}$ cells, providing a plausible mechanism for enhanced BCR signaling in Gal9-KO B cells.

Interestingly, we found that the ligands for galectin-9 are not saturated in naive B cells and raises the possibility that BCR signaling could be modified under conditions that affect the secretion of galection-9. It is currently not known if B cells secrete galectin-9, which binds to the cell surface, or if other cells secrete galectin-9, which then binds to the surface of B cells. Indeed, galectin-9 is expressed in multiple cell types, including $\mathrm{T}$ cells, natural killer cells, and monocytes ${ }^{62}$. Alterations in galectin-9 secretion and its association with cell surface proteins would provide a mechanism to adjust the threshold of B-cell activation and thus tune B-cell responses. Moreover, recent reports indicate that galectin-9 is induced under inflammatory conditions in peripheral blood mononuclear cells and mesenchymal stromal cells ${ }^{63}$, and elevated in the serum of patients in a variety of infections, including HIV, cytomegalovirus, and dengue infection ${ }^{64-66}$. How this affects the B-cell response in these infections is an important question to address.

\section{Methods}

Mice. C57BL/6 (WT) mice were obtained from Charles River, Lgals9 ${ }^{-1-}$ (Gal9KO) mice were obtained from Steven Beverley (Washington University) on behalf of The Scripps Research Institute, and Lgals1 ${ }^{-/-}$(Gall-KO) were obtained from The Jackson Laboratory. Mice were used at 2-6 months of age for all functional and biochemical experiments and were age- and sex-matched within each experiment. Mice were housed in specific pathogen-free animal facility at University of Toronto Scarborough, Toronto, Canada. All procedures were approved by the Local Animal Care Committee at the University of Toronto Scarborough.

Cell lines and culturing. A20 B cells and A20 expressing Hel-specific IgM (D1.3) were obtained from F. Batista (Ragon Institute), and Jurkat $\mathrm{T}$ cells were obtained from T. Watts (University of Toronto). Cell lines were not tested for mycoplasma contamination. Cells were maintained at $37^{\circ} \mathrm{C}$ with $5 \% \mathrm{CO}_{2}$ in RPMI 1640 containing $10 \%$ heat-inactivated fetal bovine serum (FBS), $100 \mathrm{U} / \mathrm{mL}$ penicillin and streptomycin (all from Gibco), and $50 \mu \mathrm{M} 2$-mercaptoethanol (Amresco).

Cell isolation. Splenocytes were isolated from C57BL/6 WT, Gal9-KO, and Gal1$\mathrm{KO}$ mice using $70 \mu \mathrm{m}$ cell strainer and phosphate-buffered saline (PBS, pH 7.4, Life Technologies) to collect a single-cell suspension. B cells were purified using negative isolation kit (Miltenyi Biotec Inc. or Stem Cell technologies) according to the manufacturer's protocol.

Immunohistochemistry. Inguinal lymph nodes from C57BL/6 mice were isolated and immersed in Optimal Cutting Temperature compound (TissueTek) followed by submersion in 2-methylbutane cooled in liquid nitrogen. Frozen tissue blocks were cryosectioned into $12 \mu \mathrm{m}$ slices and adhered to Superfrost Plus microscope slides (VWR), fixed in 4\% paraformaldehyde (PFA), and then air-dried. Tissue slices were rehydrated with PBS for $15 \mathrm{~min}$, permeabilized with $0.5 \%$ Tween- 20 in PBS for $5 \mathrm{~min}$, then blocked with $5 \%$ goat serum in PBS for $30 \mathrm{~min}$. Sections were incubated with CD169-FITC (clone 3D6.112, Biolegend), B220-allophycocyanin (APC) (clone RA3-6B2, Invitrogen), and goat anti-mouse galectin-9 (R\&D Systems, Cat. No. AF3535) at 1:200 v/v in blocking buffer for $1 \mathrm{~h}$ at room temperature. Sections were washed five times with $0.5 \%$ Tween-20 in PBS, and then incubated with secondary antibody Cy3-conjugated bovine anti-goat (Jackson Immunoresearch, Cat. No. 805-165-180) at 1:1000 v/v for $1 \mathrm{~h}$ at room temperature. Slides were washed five times and then mounted in Fluoro-Gel with $\mathrm{DABCO}^{\mathrm{m}}$ Antifading Mounting Medium. Slides were imaged by laser scanning confocal microscopy with Zeiss LSM510 Meta using a $\times 100 /$ numerical aperture (NA) 1.40 oilimmersion objective.

Surface staining of galectin-9 for flow cytometry and imaging. C57BL/6, Gal9$\mathrm{KO}$, or rGal9-treated B cells were first blocked with purified rat anti-mouse CD16/ 32 (Fc-block, 1:250 v/v, clone FCR-4G8, Invitrogen) in fluorescence-activated cell sorting (FACS) buffer (1\% bovine serum albumin (BSA), $4 \mathrm{mM}$ EDTA, and $0.01 \%$ $\mathrm{NaN}_{3}$ in PBS) for $30 \mathrm{~min}$ at $4{ }^{\circ} \mathrm{C}$. Cells were washed three times with FACS buffer and then immunostained with phycoerythrin-conjugated rat anti-mouse galectin- 9 antibody (clone 108A2, Biolegend) at 1:200 v/v, or stained with goat anti-galectin- 9 antibody (R\&D Systems, Cat. No. AF3535) at 1:100 v/v in FACS buffer for $30 \mathrm{~min}$ at $4{ }^{\circ} \mathrm{C}$, followed by Cy3-conjugated AffiniPure bovine anti-goat antibody (Jackson ImmunoResearch Laboratories, Cat. No. 805-165-180) at 1:1000 v/v in FACS buffer for $30 \mathrm{~min}$ at $4{ }^{\circ} \mathrm{C}$. Cells were then stained with the following antibodies: APCconjugated anti-CD19 (clone ID3, Biolegend) at 1:200 v/v; Alexa Fluor 488 or
Alexa Fluor 644 AffiniPure Fab fragment rat anti-mouse IgM, $\mu$-chain-specific (Jackson ImmunoResearch Laboratories, Cat. No 115-547-020 or Cat. No 115-607020 ) at $1: 200 \mathrm{v} / \mathrm{v}$ in FACS buffer for $30 \mathrm{~min}$ at $4{ }^{\circ} \mathrm{C}$. Cells were washed and resuspended in FACS buffer for flow analysis or imaged by spinning disc confocal microscopy. Cells were analyzed by flow cytometry (BD Fortessa) and plotted using Flowjo software (TreeStar). Cells were imaged by spinning disc confocal microscopy (Quorum Technologies) consisting of an inverted fluorescence microscope (DMI6000B; Leica) equipped with a $\times 63 / 1.4$ NA oil-immersion objective and an electron-multiplying charge-coupled device (EMCCD) camera (ImagEM; Hamamatsu). Fluorescence intensity images of galectin-9 were pseudo-colored using the fire lookup table in ImageJ. Quantification of number of puncta for all $z$-sections was performed in Image using a threshold of $2 \times$ background intensity followed by "Analyze Particles" algorithm with the following parameters: size 5-100; circularity $0.00-1.00$.

Planar lipid bilayer. Artificial planar lipid bilayers were prepared by liposome spreading ${ }^{67}$ in FCS2 chambers (Bioptechs). 1, 2-dioleoyl-sn-glycero-3-phosphocoline liposomes (Avanti Polar Lipids, Inc.) were mixed with $2.5 \times 10^{-4} \%$ biotinylated (N-Biotinyl Cap PE; Avanti Polar Lipids, Inc) liposomes. Alexa Fluor 633 streptavidin (1:1000 v/v Life Technologies, Cat. No S21375) was used to tether monobiotinylated anti-mouse kappa light chain (clone HB58, purified in house from hybridoma) as surrogate antigen. Cells were assayed in chamber buffer $(0.5 \%$ FBS, $2 \mathrm{mM} \mathrm{MgCl} 2,0.5 \mathrm{mM} \mathrm{CaCl}_{2}$, and $1 \mathrm{~g} / \mathrm{L}$ D-glucose in PBS). Freshly isolated splenocytes from WT and Gal9-KO mice were allowed to spread and form clusters for $90 \mathrm{~s}$, and then fixed with $4 \% \mathrm{PFA}$ for $15 \mathrm{~min}$ at $37^{\circ} \mathrm{C}$. Cells were imaged using a spinning disc confocal microscope as described above. Images were analyzed using Volocity (Perkin Elmer) to quantify area of cell-bilayer contact, total antigen intensity, and mean antigen intensity, and plotted using Prism6 (GraphPad). Antigen was pseudo-colored using the fire lookup table provided in ImageJ (National Institutes of Health)

Pull-down assay and mass spectrometry. The protocol was adapted from ref. ${ }^{33}$ In all, $10^{7}-5 \times 10^{7}$ purified B cells from Gal9-KO mice were lysed in NP40 lysis buffer (50 mM HEPES (pH 8.0), $150 \mathrm{mM} \mathrm{NaCl}, 5 \mathrm{mM}$ dithiothreitol (DTT), $5 \mathrm{mM}$ EDTA, $0.1 \%$ Nonidet-P40, and Roche cOmplete mini EDTA-free protease inhibitor) with three cycles of freeze/thaw using liquid nitrogen. Lysates were cleared by centrifugation at $21000 \times g$ for $10 \mathrm{~min}$ at $4{ }^{\circ} \mathrm{C}$. The cell lysate was incubated with anti-FLAG beads (Sigma-Aldrich) conjugated with $10 \mu \mathrm{g}$ FLAG-tagged rGal9 (kindly provided by M. Ostrowski, University of Toronto) for $3 \mathrm{~h}$ at $4{ }^{\circ} \mathrm{C}$. Beads were subjected to magnetic separation and washing in lysis buffer. Bound proteins were first eluted by $200 \mathrm{mM}$ lactose solution (in distilled water), followed by denaturing elution $\left(125 \mathrm{mM} \mathrm{NH}_{4} \mathrm{OH}\right)$. Denatured eluents were evaporated to dryness to remove $\mathrm{NH}_{4} \mathrm{OH}$ using a vacuum centrifuge, followed by resuspension in $50 \mathrm{mM}$ ammonium bicarbonate ( $\mathrm{pH} 8.0$ ). Both eluents were then subjected to insolution tryptic digestion followed by peptide desalting and concentration using OMIX C18 tips (Agilent) according to the manufacturer's instructions. Eluted peptides were dried by vacuum centrifugation and stored at $-20^{\circ} \mathrm{C}$ prior to analysis. Peptide separations were performed with an increasing acetonitrile gradient (5-40\% acetonitrile and $0.1 \%$ formic acid) over 90 min using an EASY nLC1000 HPLC equipped with a $10.5 \mathrm{~cm}$ C18 column coupled online to an LTQ XL mass spectrometer (Thermo). MS1 scans were acquired between 400 and $1800 \mathrm{~m} / \mathrm{z}$ and up to six of the most intense parent ions were selected for collision-induced dissociation with an isolation width of $2 \mathrm{Da}$ and normalized collision energy of 35 $\mathrm{eV}$. Parent ions selected for analysis more than three times in $30 \mathrm{~s}$ were dynamically excluded from analysis for $45 \mathrm{~s}$. Raw spectra were converted to mzXML format using msConvert (PMID: 23051804) and searched against the mouse and Escherichia coli proteomes as well as the sequence of Lgals 9 and a database of commonly observed contaminants using X! Tandem (PMID: 14976030). Parent ion tolerances were set to +3 and $-2 \mathrm{Da}$ and fragment ion mass tolerance was set to $0.4 \mathrm{Da}$. Oxidation of methionine and tryptophan, as well as deamidation of asparagine and glutamine was permitted as potential modifications during refinement. Results were further compared using ProhitsVM ${ }^{68}$.

Pull-down assay for western blotting. A total of $1-3 \times 10^{7}$ purified $\mathrm{B}$ cells from Gal9-KO mice were lysed in RIPA buffer (10 mM Tris- $\mathrm{HCl}$ (pH 8.0), 1 mM EDTA, $1 \%$ Triton X-100, $0.1 \%$ sodium deoxycholate, $0.1 \%$ SDS, $140 \mathrm{mM} \mathrm{NaCl}$, and $1 \mathrm{mM}$ phenylmethylsulfonyl fluoride (PMSF)). Lysates were cleared by centrifugation at $21000 \times g$ for $10 \mathrm{~min}$ at $4^{\circ} \mathrm{C}$. Cell lysates were incubated with anti-FLAG beads (Sigma-Aldrich) conjugated with $10 \mu \mathrm{g}$ FLAG-tagged rGal9 for $3 \mathrm{~h}$ at $4{ }^{\circ} \mathrm{C}$ in the presence or absence of $50 \mathrm{mM}$ D-lactose. Beads were subjected to magnetic separation and washed in RIPA buffer. Bound proteins were eluted using $200 \mathrm{mM}$ D-lactose in PBS. Protein was mixed with equal volume of $2 \times$ Laemmli sample buffer, heated to $95^{\circ} \mathrm{C}$ for $10 \mathrm{~min}$, and separated by SDS-polyacrylamide gel electrophoresis (SDS-PAGE). Proteins were transferred to polyvinylidene fluoride (PVDF) membrane and blocked with 5\% non-fat milk in TBST (20 nM Tris ( $\mathrm{pH} 7.5), 150 \mathrm{mM} \mathrm{NaCl}$, and $0.1 \%$ Tween-20) for $30 \mathrm{~min}$ at room temperature with gentle shaking. Primary antibodies rat anti-mouse CD45RA (clone 30-F11; BioLegend) and goat anti-mouse IgM (Jackson Immunoresearch, Cat. No. 115-005020 ) were incubated at 1:1000 overnight at $4^{\circ} \mathrm{C}$ with gentle shaking. Secondary 
antibodies horseradish peroxidase (HRP)-conjugated donkey anti-rat and donkey anti-goat (Abcam, Cat. No ab102182 and ab97110) were incubated at 1:10 000 in $5 \%$ non-fat milk in TBST for $1 \mathrm{~h}$ at room temperature with gentle shaking. Membranes were washed with TBST and developed with chemiluminescent substrate (West Pico, Thermo Fisher), and imaged by ChemiDoc (Bio-Rad).

Far-western blotting. In all, $1-5 \times 10^{7}$ purified primary murine B cells, D1.3 B cells, A20 B cells, or Jurkat T cells were lysed in RIPA buffer $(10 \mathrm{mM}$ Tris- $\mathrm{HCl} \mathrm{pH}$ 8.0; $1 \mathrm{mM}$ EDTA, $1 \%$ Triton X-100, $0.1 \%$ sodium deoxycholate, $0.1 \%$ SDS, $140 \mathrm{mM}$ $\mathrm{NaCl}$, and $1 \mathrm{mM}$ PMSF). Lysates were cleared by centrifugation at $21000 \times g$ for $10 \mathrm{~min}$ at $4^{\circ} \mathrm{C}$. A unit of $25 \mu \mathrm{g}$ of whole-cell lysate was resolved by $10 \%$ SDS-PAGE and transferred to PVDF membrane. Proteins were re-natured in decreasing concentrations of guanidine- $\mathrm{HCl}(6-0 \mathrm{M})$ according to ref. ${ }^{34}$. Membranes were then blocked with $5 \%$ non-fat milk in TBST for $30 \mathrm{~min}$ at room temperature with gentle shaking. A unit of $5 \mu \mathrm{g} / \mathrm{mL}$ FLAG-tagged-recombinant galectin- 9 in $2.5 \%$ skim milk in TBST was used as a bait protein, blots were incubated with bait protein overnight at $4{ }^{\circ} \mathrm{C}$ with gentle shaking. Blots were then washed and incubated with anti-FLAG (clone M2, Sigma-Aldrich) at 1:1000 in 5\% skim milk in TBST for $1 \mathrm{~h}$ at room temperature. Blots were washed and incubated with HRPconjugated anti-mouse antibody (Jackson Immunoresearch, Cat. No. 115-035-003) at 1:10 000 in $5 \%$ non-fat milk in TBST for $1 \mathrm{~h}$ at room temperature with gentle shaking. Membranes developed with chemiluminescent substrate (West Pico, Thermo Fisher) and imaged by ChemiDoc (Bio-Rad).

rGal9 and Fab fragment labeling for single-particle tracking. Mouse rGal9 (R\&D Systems) was reconstituted at $0.2 \mathrm{mg} / \mathrm{mL}$ in $0.1 \mathrm{M} \mathrm{NaHCO} 3$ and incubated with $0.2 \mathrm{mg} / \mathrm{mL}$ Alexa Fluor 555 NHS Ester for $1 \mathrm{~h}$ at room temperature with gentle mixing. Following labeling, the mixture was dialyzed against $20 \mathrm{mM}$ MOPS, $500 \mathrm{mM}$ sodium chloride, $0.5 \mathrm{mM}$ EDTA, and $1 \mathrm{mM}$ DTT using a 10000 molecular weight cutoff Slide-A-Lyzer ${ }^{\circ}$ Dialysis Cassette (Thermo Scientific). A volume of $20 \mu \mathrm{l}$ of $1 \mathrm{M} \mathrm{NaHCO} 3$ was added to $200 \mu \mathrm{l}$ of $1 \mathrm{mg} / \mathrm{mL}$ goat anti-mouse IgM, $\mu$ chain-specific (Jackson ImmunoResearch, Cat. No. 115-007-020) and incubated with $40 \mu \mathrm{g} / \mathrm{mL}$ Attotec ${ }^{\circ} 633$ NHS Ester for $1 \mathrm{~h}$ at room temperature with gentle mixing. Following labeling, the mixture was dialyzed against PBS.

\section{Glass coverslip coating for single-particle tracking. Glass coverslips were} cleaned in chromic acid (Fisher) for $20 \mathrm{~min}$ followed by rinsing with water and acetone. Coverslips were air-dried and then incubated with $1 \mu \mathrm{g} / \mathrm{mL}$ anti-MHC class II (clone M5/114, Sunnybrook Research Institute) for $2 \mathrm{~h}$ at room temperature and then washed with PBS.

Cell labeling for single-particle tracking. Primary murine B cells from WT and Gal9-KO mice were labeled with $4 \mathrm{ng} / \mathrm{mL}$ Attotec ${ }^{\circ}$ 633-labeled goat anti-mouse IgM Fab fragment (Jackson ImmunoResearch, Cat. No. 115-007-020) in 0.5\% FBS in PBS for $15 \mathrm{~min}$ at $4{ }^{\circ} \mathrm{C}$. For rGal9 treatment, $5 \times 10^{6} \mathrm{~B}$ cells from Gal9-KO mice were incubated with $0.5 \mu \mathrm{M}$ labeled rGal9 mixed with $0.5 \mu \mathrm{M}$ non-labeled rGal9 in complete media for $30 \mathrm{~min}$ at $37^{\circ} \mathrm{C}$. Labeled cells were washed twice with PBS and resuspended in chamber buffer. Labeled cells were stored on ice prior to imaging. Just before imaging, cells were incubated at $37^{\circ} \mathrm{C}$ for $5 \mathrm{~min}$.

Instrument for single-particle tracking and dSTORM. Single-molecule fluorescence microscopy was performed with a TIRF microscope (Quorum Technologies) based on an inverted microscope (DMI6000B; Leica), HCX PL APO ×100/1.47 oilimmersion objective, and Evolve Delta EMCCD camera (Photometrics). Images were acquired continuously at 20 frames/s for $10 \mathrm{~s}$ with an EM gain of 200 and the exposure time of $50 \mathrm{~ms}$.

Single-particle tracking. We used the well-established single-particle tracking algorithm by Crocker and Grier ${ }^{69}$ implemented in Matlab (The MathWorks) by Daniel Blair and Eric Dufresne (http://physics.georgetown.edu/matlab/). Particle positions were determined by measuring the the centroid position with sub-pixel accuracy based on the local maxima and then linking particle positions. Track lengths $<10$ were discarded in order to reduce statistical noise. Diffusion coefficients for individual trajectories were determined form the first three data points of the mean squared displacement curves of each trajectory.

Sample preparation for dSTORM. Primary B cells from WT and Gal9-KO mice were stained with Alexa Fluor 647-conjugated Fab fragment goat anti-mouse IgM, $\mu$-chain-specific (Jackson ImmunoResearch, Cat. No. 115-607-020) at $1.5 \mu \mathrm{g} / \mathrm{mL}$ in PBS containing $2 \% \mathrm{FBS}$ for $15 \mathrm{~min}$ at $4{ }^{\circ} \mathrm{C}$, then washed twice with PBS. For dualcolor dSTORM, primary WT and Gal9-KO B cells were stained with Alexa Fluor 488-conjugated Fab fragment goat anti-mouse IgM, $\mu$-chain-specific (Jackson ImmunoResearch, Cat. No 115-547-020) at $2.8 \mu \mathrm{g} / \mathrm{mL}$ and either Alexa Fluor 647 anti-mouse CD22 (OX-97, Biolegend) at $2.5 \mu \mathrm{g} / \mathrm{mL}$ or Alexa Fluor 647 anti-mouse/ human B220 (RA3-6B2, Biolegend) at $2.5 \mu \mathrm{g} / \mathrm{mL}$ all in PBS containing $2 \%$ FBS for $20 \mathrm{~min}$ at $4{ }^{\circ} \mathrm{C}$, then washed twice with PBS. Cells were allowed to spread on antiMHC-class II-coated coverslips for $10 \mathrm{~min}$ at $37^{\circ} \mathrm{C}$. Coverslips were washed gently with PBS to remove unbound cells. Cells were fixed with fixation solution (4\% PFA and $0.2 \%$ glutaraldehyde in PBS) for $40 \mathrm{~min}$ at room temperature. The coverslips were washed three times with PBS. Before single-color dSTORM imaging, samples were incubated in PBS containing $0.1 \mathrm{M}$ mercaptoethylamine, $0.5 \mathrm{mg} / \mathrm{mL}$ glucose oxidase, $40 \mu \mathrm{g} / \mathrm{mL}$ catalase, and $10 \%$ glucose. For dual-color dSTORM, samples were incubated in PBS containing $0.1 \mathrm{M} \beta$-mercaptoethylamine hydrochloride (Sigma-Aldrich), 3\% (v/v) OxyFlour ${ }^{\text {Dit }}$ (Oxyrase Inc., Mansfield, Ohio, USA), 20\% (v/v) of sodium DL-lactate solution (L1375, Sigma-Aldrich) adjusted to $\mathrm{pH} \sim 8.3$. Fiducial markers (100 nm TetraSpeck Fluorescent Microspheres, Invitrogen) were added to buffer and allowed to settle for $5 \mathrm{~min}$ prior to imaging.

dSTORM acquisition and image reconstruction. ASTORM images were acquired on a TIRF microscope as described above. For Alexa Fluor ${ }^{\circledR}$ 647, photoconversion was achieved with $633-\mathrm{nm}$ laser (intensity ranged from 80 to $100 \mathrm{~mW} / \mathrm{cm}^{2}$ ) illumination and conversion from the dark state with 488 -nm laser illumination (intensity range from 5 to $20 \mathrm{~mW} / \mathrm{cm}^{2}$ ). Ten thousand images were acquired at a frame rate of 33 frames/s. Dual-color dSTORM images were acquired sequentially on a TIRF microscope, first imaging Alexa 647 followed by Alexa 488 to prevent photobleaching of Alexa 647. For Alexa Fluor ${ }^{\circledast} 647$, photoconversion was achieved with $633-\mathrm{nm}$ laser (intensity ranged from 80 to $100 \mathrm{~mW} / \mathrm{cm}^{2}$ ) illumination and conversion from the dark state with $402-\mathrm{nm}$ laser illumination (intensity range from 5 to $20 \mathrm{~mW} / \mathrm{cm}^{2}$ ). For Alexa Fluor ${ }^{-6} 488$, photoconversion was achieved with 488 -nm laser (intensity ranged from 80 to $100 \mathrm{~mW} / \mathrm{cm}^{2}$ ). Eight thousand images were acquired at a frame rate of 30 frames/s. Fiducial markers, which were visible in both the 488- and 647-nm channels, were used to align the two channels. The images of the beads in both channels were used to calculate a polynomial transformation function that mapped the 488-nm channel onto the $647-\mathrm{nm}$ channel, using the MultiStackReg plugin of ImageJ. The transformation matrix was applied to each frame of the 488-nm channel stack. Reconstructed images were acquired using ThunderSTORM plugin for Image ${ }^{70}$ according to the parameters shown in Supplementary Fig. 8A. Briefly, camera setup was as follows: pixel size $(101.5 \mathrm{~nm})$; photoelectron per A/D count 2.4; base level [A/D count] 414; and an EM gain of 50 (dSTORM) or 200 (dual-color dSTORM). Image filtering was applied to remove camera noise and enhance photoswitching events using a wavelet filter (B-Spline) with B-Spline order of 3 and B-Spline scale of 2.0. Approximate localization of molecules was detected by local maximum method with a peak intensity threshold of std (Wave.F1) and a connectivity of 8-neighborhood. Sub-pixel localization of molecules was identified by fitting point spread function to an integrated Gaussian using weighted least squares method with a fitting radius of 3 pixels. Single molecules may not be adequately resolved in spatially dense organizations and thereby multiple activated molecules are detected as a single blob. Multiple emitter fitting analysis was used to estimate the number of molecules detected as a single blob with maximum five molecules per fitting region. To improve the multi-emitter fitting algorithm, molecules are fitted assuming the same intensity. Superresolution images were rendered with a pixel size of $20 \mathrm{~nm}$.

dSTORM post-processing, analysis, and quantification. Reconstructed dSTORM images were post-processed with drift correction using the built-in method in the ThunderSTORM plugin. The workflow for post-processing steps is shown in Supplementary Fig. 8B and consisted of the following steps: (1) remove duplicates, in which repeated localizations of single molecules in one frame, which may occur when using multiple emitter, were removed based on uncertainty radius of localization; (2) filter, in which localizations with an uncertainty $>20 \mathrm{~nm}$ were eliminated; (3) density filter, in which isolated localizations were removed based on the parameter of at least two neighbors are required in $50 \mathrm{~nm}$ radius for a localization to be accepted; (4) drift correction, in which image drift was corrected using fiducial markers; and (5) merging, in which molecules that appeared within $20 \mathrm{~nm}$ in multiple frames were merged together. The number of localizations across cells and conditions was similar (Supplementary Fig. 8C). Performance evaluation of dSTORM analysis and post-processing parameters was performed (Supplementary Methods; Supplementary Fig. 9). To reduce processing time, 4000 frames were processed for cluster analysis. For each reconstructed image of WT and Gal9-KO B cells, a $3 \times 3 \mu \mathrm{m}$ region was manually selected but excluded from the cell boundary. For each reconstructed image of rGal9-treated Gal9-KO B cells, a $3 \times 3 \mu \mathrm{m}$ region that colocalized with Gal9, was manually selected for analysis. The Hopkins index and Ripley's $H$ function analysis were performed by SuperCluster, an analysis tool kindly provided by the University of New Mexico's Spatio Temporal Modeling Center (http://stmc.unm.edu/). Cluster analysis was performed by a Bayesian, a model-based approach ${ }^{38}$. In brief, uncertainty, $x$ and $y$ coordinates of each localization in post-processed reconstructed images were exported. The selected $3 \times 3$ $\mu \mathrm{m}$ regions were analyzed by the published $\mathrm{R}$ code of Bayesian cluster analysis ${ }^{38}$ with an alpha value of 20 , pbackground of 0.5 , rseq of $(5,200,10)$, and thseq of $(0$, $50,5)$. The analyzed data were post-processed to extract data about percentage of molecules localizing in clusters, cluster radius, number of clusters, and number of molecules per clusters.

CBC analysis. Colocalization of dual dSTORM data was conducted using CBC analysis ${ }^{49}$ using ThunderSTORM. Briefly, this method uses coordinate information of each molecule instead of an intensity-based approach, which would depend on the reconstruction and post-processing parameters chosen. Furthermore, this 
method takes into account the spatial distribution of each set of localizations to prevent false positive colocalization values that result when one of the molecular species is randomly organized. First, the spatial distribution function of the neighboring localizations from the same species in each channel are calculated. Then, from the individual distribution functions, a correlation coefficient is calculated and weighted by distance to the nearest neighbor of the localization's respective species. As a result, each single molecule of each species is attributed an individual colocalization value, which provides information on the molecule's local environment. $\mathrm{CBC}$ algorithm was applied to $x-y$ coordinate lists of localizations in the $3 \times 3 \mu \mathrm{m}$ region of 647 and 488 channels. A search radius of $70 \mathrm{~nm}$, based on the radius IgM nanoclusters, was used to calculate degree of colocalization values varying from -1 (perfectly excluded) to +1 (perfectly colocalized).

Cell stimulation for western blot. Twenty-four-well plates were coated with $5 \mu \mathrm{g} /$ $\mathrm{mL}$ AffiniPure $\mathrm{F}\left(\mathrm{ab}^{\prime}\right) 2$ fragment goat anti-mouse IgM, $\mu$-chain-specific (Jackson ImmunoResearch Laboratories, Cat. No 115-006-020) in PBS overnight at $4{ }^{\circ} \mathrm{C}$. Primary naive splenic B cells from WT and Gal9-KO mice were stimulated for the indicated time, then lysed with Laemmli buffer containing $100 \mu \mathrm{M}$ DTT followed by SDS-PAGE. For rGal9 treatment, Gal9-KO or WT B cells were pre-treated with 0.1 or $1 \mu \mathrm{M}$, respectively, with recombinant mouse galectin-9 (rGal9, E. coliderived, R\&D Systems) in RPMI containing 1\% FBS (both Gibco) and incubated for $30 \mathrm{~min}$ at $37^{\circ} \mathrm{C}$. Cells were then washed once with RPMI and then stimulated as described above. Proteins were transferred to PVDF and blocked with 5\% nonfat skimmed milk or 5\% BSA in TBST for $1 \mathrm{~h}$ at room temperature or overnight (for $4 \mathrm{G} 10$ ) at $4{ }^{\circ} \mathrm{C}$ with shaking. Membranes were immunoblotted with the following antibodies overnight or for $3 \mathrm{~h}$ (for $4 \mathrm{G} 10$ ) at $4{ }^{\circ} \mathrm{C}$ : mouse anti- $\beta$-tubulin (Sigma, 1:5000 in 1\% BSA/TBST); rabbit anti-phospho-p44/42 MAPK (ERK1/2 Cell Signaling, Cat. No. 9101, 1:1000 in 1\% BSA/TBST); rabbit anti-ERK p44/42 MAPK (ERK1/2 Cell Signaling, Cat. No. 9102); 4G10 (Sunnybrook Research Institute, $1: 1000$ in $1 \% \mathrm{BSA}_{1} \% \mathrm{NaN}_{3} / \mathrm{TBST}$ ); phospho-CD19 (Tyr531 Cell Signaling, Cat. No. $3571,1: 1000$ in $1 \% \mathrm{BSA} / 1 \% \mathrm{NaN}_{3} / \mathrm{TBST}$ ); and phospho-Akt (Tyr308 Cell Signaling, Cat. No. 4056, 1:1000 in $1 \% \mathrm{BSA} / 1 \% \mathrm{NaN}_{3} / \mathrm{TBST}$ ). HRPconjugated secondary antibodies were used at 1:10 $000 \mathrm{v} / \mathrm{v}$ in $5 \%$ non-fat skimmed milk in TBST or $1 \%$ BSA in TBST and incubated for $2 \mathrm{~h}$ with shaking at room temperature. Membranes were incubated with SuperSignal (Thermo Fisher Scientific) chemiluminescent substrate and then imaged by ChemiDoc (Bio-Rad). Densitometric analysis of western blots was performed using ImageJ (NIH). The amount of phosphorylated protein was normalized to loading control and then expressed as a fold change relative to the $0 \mathrm{~min}$ time point. Full blots for all cropped blots presented in the figures are shown in Supplementary Fig. 10.

Calcium signaling. Intracellular $\mathrm{Ca}^{2+}$ flux was measured by flow cytometry. Freshly isolated splenocytes $\left(5 \times 10^{6}\right.$ cells/sample $)$ were labeled with $1 \mu \mathrm{M}$ Fluo- 4 , AM

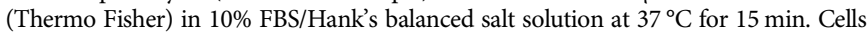
were incubated with anti-CD45R (B220) (clone RA3-6B2; Biolegend) for $5 \mathrm{~min}$ at $4{ }^{\circ} \mathrm{C}$ at a dilution of $1: 200$. Cells were washed $2 \times$ with PBS and resuspended in RPMI. After collecting a baseline reading for $30 \mathrm{~s}, 20 \mu \mathrm{g} / \mathrm{mL}$ anti-mouse kappa immunoglobulin light chain (clone HB58) or $1 \mu \mathrm{M}$ rGal9 was added to the facs tube, and change in fluorescence intensity was recorded on a Fortessa cytometer (BD Biosciences) and plotted using Flowjo software (TreeStar). Fold change was calculated by dividing the fluorescence intensity at each time point by baseline intensity.

rGal9 treatment and confocal imaging. Eight-well Lab-Tek chambers were coated with $1 \mu \mathrm{g} / \mathrm{mL}$ anti-mouse MHC II (M5/114, Sunnybrook Research Institute) antibody in PBS for $2 \mathrm{~h}$ at room temperature. In all, $5 \times 10^{6}$ primary murine B cells from WT mice were treated with $1 \mu \mathrm{M}$ recombinant galectin-9 in 2\% FBS in RPMI for $30 \mathrm{~min}$ at $37^{\circ} \mathrm{C}$. Cells were washed $1 \times$ with PBS. Cells were resuspended in PBS and allowed to adhere to chambers for $15 \mathrm{~min}$. Cells were fixed in 2\% PFA for 10 min and then washed $2 \times$ in FACS buffer (PBS, $1 \%$ BSA, and $0.1 \% \mathrm{NaN}_{3}$ ). Cells were incubated with $2 \mu \mathrm{g} / \mathrm{mL}$ purified rat anti-mouse CD16/32 (clone $2.4 \mathrm{G} 2$, BDPharmingen) in PBS for $15 \mathrm{~min}$ at $4^{\circ} \mathrm{C}$. Cells were incubated with $1 \mu \mathrm{g} / \mathrm{mL}$ goat anti-mouse Gal9 (R\&D systems, Cat. No. AF3535) in FACS buffer for $1 \mathrm{~h}$ at $4^{\circ} \mathrm{C}$. Cells were washed $3 \times$ with FACS buffer. If cells were stained to visualize CD16/32, cells were incubated with $1 \mu \mathrm{g} / \mathrm{mL}$ Alexa-Fluor594 Donkey Anti-Rat IgG (Invitrogen Cat. No A-21209) for $1 \mathrm{~h}$ at $4{ }^{\circ} \mathrm{C}$. Cells were washed $2 \times$ with PBS. Cells were incubated with $1 \mu \mathrm{g} / \mathrm{mL}$ Cy3-conjugated bovine anti-goat IgG (Jackson ImmunoResearch, Cat. No. 805-165-180) or Alexa Fluor 488 donkey anti-goat IgG secondary (Jackson ImmunoResearch, Cat. No. 705-545-147) for $1 \mathrm{~h}$ at $4{ }^{\circ} \mathrm{C}$. Cells were washed $3 \times$ with PBS. Cells were stained with fluorochrome-conjugated antibodies specific for CD45/B220 (RA3-6B2, BD Biosciences), CD22 (OX-97, Biolegend), CD19 (1D3, Biolegend), IgD (11-26c.2a, Biolegend) at 1:100 v/v or IgM (Jackson ImmunoResearch) at $1: 1000 \mathrm{v} / \mathrm{v}$ for $1 \mathrm{~h}$ at $4{ }^{\circ} \mathrm{C}$. Cells were washed $3 \times$ with PBS. Cells were mounted in Fluoro-Gel with DABCO ${ }^{\mathrm{TM}}$ (Electron Microscopy). Chambers were imaged by spinning disk confocal microscopy (Quorum Technologies) consisting of an inverted fluorescence microscope (DMI6000B; Leica) equipped with a $\times 63 / 1.4 \mathrm{NA}$ oil-immersion objective and an EMCCD camera (Image EM; Hamamatsu). Images were acquired using Metamorph software (Molecular Devices). Images were analyzed using Volocity software (Perkin Elmer). The fluorescence signal of CD45, CD22, CD19, IgM, IgD, or Fc $\gamma$ RIIb was used to define a mask delineating the membrane region. Galghigh regions were determined by the fluorescence signal of Gal9. Galglow regions were determined by subtracting the Galghigh regions from the membrane region. The mean florescence intensity of CD45, CD22, CD19, IgD, FcyRIIb, and IgM was calculated in Volocity.

Lipid raft staining. Primary murine WT B cells and $1 \mu \mathrm{M}$ rGal9-treated B cells were labeled with $1 \mu \mathrm{g} / \mathrm{mL}$ Alexa Fluor 555-conjugated Cholera toxin subunit B (Vybrant $^{\mathrm{m} \mathrm{m}}$ Alexa Fluor ${ }^{\mathrm{max}} 555$ Lipid Raft Labeling Kit, Thermo Fisher Scientific) for $15 \mathrm{~min}$ on ice. Cells were washed $2 \times$ with PBS for $5 \mathrm{~min}$ at $4^{\circ} \mathrm{C}$. Cells were then allowed to adhere to anti-MHC II-coated Mattek chambers for $10 \mathrm{~min}$. Cells were fixed with 2\% PFA for $15 \mathrm{~min}$ and washed $3 \times$ with PBS. Cells were then stained for specific proteins as described above.

B-cell apoptosis. Freshly isolated naive B cells from WT and Gal9-KO mice were treated with $1 \mu \mathrm{M}$ recombinant mouse galectin-9 (rGal9, E. coli-derived, R\&D Systems) for $30 \mathrm{~min}$ at $37^{\circ} \mathrm{C}$, followed by staining with Fluorescein isothiocyanate (FITC)-conjugated Annexin V and propidium iodide according to the manufacturer's protocol (Annexin V Apoptosis Detection Kit, eBioscience), and analyzed by flow cytometry (BD Fortessa). Data were analyzed using FlowJo software.

Statistical analysis. Statistical analysis was performed using GraphPad Prism. The distribution of data was tested using D'Agostino-Pearson omnibus normality test. Comparisons between two groups were performed using Mann-Whitney for data with non-normal distribution. Comparisons between multiple groups were performed by ordinary one-way analysis of variance (ANOVA) for data with normal distribution and Kruskal-Wallis test for data with non-normal distribution. For post hoc analysis, Tukey's multiple comparison was used for normally distributed data and Dunn's multiple comparisons test was used for non-normally distributed data. Western blot data were analyzed by two-way ANOVA followed by Sidak's multiple comparisons test. Sample sizes were chosen based on prior literature using similar methods and reporting moderate effect size. Estimate of variance is reported as standard error of the mean for each data set.

Code availability. ThunderStorm plugin for ImageJ is available at http://zitmen. github.io/thunderstorm/. SuperCluster is available from the University of New Mexico's Spatio Temporal Modeling Center (http://stmc.unm.edu/). Bayesian cluster analysis code is available in supplementary information in ref. ${ }^{38}$. Singleparticle tracking algorithms implemented in Matlab (The MathWorks) is available at http://physics.georgetown.edu/matlab/.

Data availability. Lgals9-/- mice require an MTA from The Scripps Research Institute (TSRI). The data that support the findings of this study are available from the corresponding author upon reasonable request.

Received: 13 December 2017 Accepted: 26 July 2018

Published online: 17 August 2018

\section{References}

1. Kurosaki, T., Shinohara, H. \& Baba, Y. B cell signaling and fate decision. Annu. Rev. Immunol. 28, 21-55 (2010).

2. Depoil, D. et al. CD19 is essential for B cell activation by promoting B cell receptor-antigen microcluster formation in response to membrane-bound ligand. Nat. Immunol. 9, 63-72 (2008).

3. Choudhuri, K. \& Dustin, M. L. Signaling microdomains in T cells. FEBS Lett. 584, 4823-4831 (2010).

4. Harwood, N. E. \& Batista, F. D. New insights into the early molecular events underlying B cell activation. Immunity 28, 609-619 (2008).

5. Doh, J. \& Irvine, D. J. Immunological synapse arrays: patterned protein surfaces that modulate immunological synapse structure formation in $\mathrm{T}$ cells. Proc. Natl Acad. Sci. USA 103, 5700-5705 (2006).

6. Mossman, K. D., Campi, G., Groves, J. T. \& Dustin, M. L. Altered TCR signaling from geometrically repatterned immunological synapses. Science 310, 1191-1193 (2005).

7. Salaita, K. et al. Restriction of receptor movement alters cellular response: physical force sensing by EphA2. Science 327, 1380-1385 (2010).

8. Lillemeier, B. F., Pfeiffer, J. R., Surviladze, Z., Wilson, B. S. \& Davis, M. M. Plasma membrane-associated proteins are clustered into islands attached to the cytoskeleton. Proc. Natl Acad. Sci. USA 103, 18992-18997 (2006).

9. Mattila, P. K. et al. The actin and tetraspanin networks organize receptor nanoclusters to regulate B cell receptor-mediated signaling. Immunity 38, 461-474 (2013)

10. Maity, P. C. et al. B cell antigen receptors of the IgM and IgD classes are clustered in different protein islands that are altered during B cell activation. Sci. Signal. 8, ra93 (2015). 
11. Andrews, N. L. et al. Actin restricts FcepsilonRI diffusion and facilitates antigen-induced receptor immobilization. Nat. Cell Biol. 10, 955-963 (2008).

12. Treanor, B. et al. The membrane skeleton controls diffusion dynamics and signaling through the B cell receptor. Immunity 32, 187-199 (2010).

13. Charrier, C., Ehrensperger, M. V., Dahan, M., Levi, S. \& Triller, A. Cytoskeleton regulation of glycine receptor number at synapses and diffusion in the plasma membrane. J. Neurosci. 26, 8502-8511 (2006).

14. Douglass, A. D. \& Vale, R. D. Single-molecule microscopy reveals plasma membrane microdomains created by protein-protein networks that exclude or trap signaling molecules in T cells. Cell 121, 937-950 (2005).

15. Tolar, P., Hanna, J., Krueger, P. D. \& Pierce, S. K. The constant region of the membrane immunoglobulin mediates $\mathrm{B}$ cell-receptor clustering and signaling in response to membrane antigens. Immunity 30, 44-55 (2009).

16. Yang, J. \& Reth, M. Oligomeric organization of the B-cell antigen receptor on resting cells. Nature 467, 465-469 (2010).

17. Owen, D. M., Williamson, D. J., Magenau, A. \& Gaus, K. Sub-resolution lipid domains exist in the plasma membrane and regulate protein diffusion and distribution. Nat. Commun. 3, 1256 (2012).

18. Brewer, C. F., Miceli, M. C. \& Baum, L. G. Clusters, bundles, arrays and lattices: novel mechanisms for lectin-saccharide-mediated cellular interactions. Curr. Opin. Struct. Biol. 12, 616-623 (2002).

19. Garner, O. B. \& Baum, L. G. Galectin-glycan lattices regulate cell-surface glycoprotein organization and signalling. Biochem. Soc. Trans. 36, 1472-1477 (2008).

20. Lajoie, P., Goetz, J. G., Dennis, J. W. \& Nabi, I. R. Lattices, rafts, and scaffolds: domain regulation of receptor signaling at the plasma membrane. J. Cell Biol. 185, 381-385 (2009)

21. Rabinovich, G. A., Toscano, M. A., Jackson, S. S. \& Vasta, G. R. Functions of cell surface galectin-glycoprotein lattices. Curr. Opin. Struct. Biol. 17, 513-520 (2007).

22. Demetriou, M., Granovsky, M., Quaggin, S. \& Dennis, J. W. Negative regulation of T-cell activation and autoimmunity by Mgat5 $\mathrm{N}$-glycosylation. Nature 409, 733-739 (2001).

23. Espeli, M., Mancini, S. J. C., Breton, C., Poirier, F. \& Schiff, C. Impaired B-cell development at the pre-BII-cell stage in galectin-1-deficient mice due to inefficient pre-BII/stromal cell interactions. Blood 113, 5878-5886 (2009).

24. Ungerer, C. et al. Galectin-9 is a suppressor of T and B cells and predicts the immune modulatory potential of mesenchymal stromal cell preparations. Stem Cells Dev. 23, 755-766 (2014).

25. Tureci, O., Schmitt, H., Fadle, N., Pfreundschuh, M. \& Sahin, U. Molecular definition of a novel human galectin which is immunogenic in patients with Hodgkin's disease. J. Biol. Chem. 272, 6416-6422 (1997).

26. Zhu, C. et al. The Tim-3 ligand galectin- 9 negatively regulates $\mathrm{T}$ helper type 1 immunity. Nat. Immunol. 6, 1245-1252 (2005).

27. Seki, M. et al. Galectin-9 suppresses the generation of Th17, promotes the induction of regulatory $\mathrm{T}$ cells, and regulates experimental autoimmune arthritis. Clin. Immunol. 127, 78-88 (2008).

28. Harwood, N. E. \& Batista, F. D. Antigen presentation to B cells. F1000 Biol. Rep. 2, 87 (2010)

29. Fleire, S. J. et al. B cell ligand discrimination through a spreading and contraction response. Science 312, 738-741 (2006).

30. Bi, S., Earl, L. A., Jacobs, L. \& Baum, L. G. Structural features of galectin-9 and galectin-1 that determine distinct T cell death pathways. J. Biol. Chem. 283, 12248-12258 (2008).

31. Kashio, Y. et al. Galectin-9 induces apoptosis through the calcium-calpaincaspase-1 pathway. J. Immunol. 170, 3631-3636 (2003).

32. Wada, J., Ota, K., Kumar, A., Wallner, E. I. \& Kanwar, Y. S. Developmental regulation, expression, and apoptotic potential of galectin-9, a beta-galactoside binding lectin. J. Clin. Invest. 99, 2452-2461 (1997).

33. Clayton, K. L. et al. T cell Ig and mucin domain-containing protein 3 is recruited to the immune synapse, disrupts stable synapse formation, and associates with receptor phosphatases. J. Immunol. 192, 782-791 (2014).

34. Wu, Y., Li, Q. \& Chen, X. Z. Detecting protein-protein interactions by Far western blotting. Nat. Protoc. 2, 3278-3284 (2007).

35. Huang, B., Jones, S. A., Brandenburg, B. \& Zhuang, X. Whole-cell 3D STORM reveals interactions between cellular structures with nanometer-scale resolution. Nat. Methods 5, 1047-1052 (2008).

36. Lee, J., Sengupta, P., Brzostowski, J., Lippincott-Schwartz, J. \& Pierce, S. K. The nanoscale spatial organization of B-cell receptors on immunoglobulin Mand G-expressing human B-cells. Mol. Biol. Cell 28, 511-523 (2017).

37. Coltharp, C., Yang, X. \& Xiao, J. Quantitative analysis of single-molecule superresolution images. Curr. Opin. Struct. Biol. 28, 112-121 (2014).

38. Griffie, J. et al. A Bayesian cluster analysis method for single-molecule localization microscopy data. Nat. Protoc. 11, 2499-2514 (2016).

39. Treanor, B., Depoil, D., Bruckbauer, A. \& Batista, F. D. Dynamic cortical actin remodeling by ERM proteins controls BCR microcluster organization and integrity. J. Exp. Med. 208, 1055-1068 (2011).
40. Hermiston, M. L., Xu, Z. \& Weiss, A. CD45: a critical regulator of signaling thresholds in immune cells. Annu. Rev. Immunol. 21, 107-137 (2003).

41. Shrivastava, P., Katagiri, T., Ogimoto, M., Mizuno, K. \& Yakura, H. Dynamic regulation of Src-family kinases by CD45 in B cells. Blood 103, 1425-1432 (2004).

42. Muller, J. \& Nitschke, L. The role of CD22 and Siglec-G in B-cell tolerance and autoimmune disease. Nat. Rev. Rheumatol. 10, 422-428 (2014).

43. Leprince, C., Draves, K. E., Geahlen, R. L., Ledbetter, J. A. \& Clark, E. A. CD22 associates with the human surface IgM-B-cell antigen receptor complex. Proc. Natl Acad. Sci. USA 90, 3236-3240 (1993).

44. Zhang, M. \& Varki, A. Cell surface sialic acids do not affect primary CD22 interactions with CD45 and surface IgM nor the rate of constitutive CD22 endocytosis. Glycobiology 14, 939-949 (2004).

45. Doody, G. M. et al. A role in B cell activation for CD22 and the protein tyrosine phosphatase SHP. Science 269, 242-244 (1995).

46. Cheng, P. C., Dykstra, M. L., Mitchell, R. N. \& Pierce, S. K. A role for lipid rafts in B cell antigen receptor signaling and antigen targeting. J. Exp. Med. 190, 1549-1560 (1999).

47. Eleme, K. et al. Cell surface organization of stress-inducible proteins ULBP and MICA that stimulate human NK cells and T cells via NKG2D. J. Exp. Med. 199, 1005-1010 (2004).

48. Gupta, N. et al. Quantitative proteomic analysis of B cell lipid rafts reveals that ezrin regulates antigen receptor-mediated lipid raft dynamics. Nat. Immunol. 7, 625-633 (2006)

49. Malkusch, S. et al. Coordinate-based colocalization analysis of singlemolecule localization microscopy data. Histochem. Cell Biol. 137, 1-10 (2012).

50. Gasparrini, F. et al. Nanoscale organization and dynamics of the siglec CD22 cooperate with the cytoskeleton in restraining BCR signalling. EMBO J. 35, 258-280 (2016)

51. Garcia-Parajo, M. F., Cambi, A., Torreno-Pina, J. A., Thompson, N. \& Jacobson, K. Nanoclustering as a dominant feature of plasma membrane organization. J. Cell Sci. 127, 4995-5005 (2014).

52. Torreno-Pina, J. A. et al. Enhanced receptor-clathrin interactions induced by $\mathrm{N}$-glycan-mediated membrane micropatterning. Proc. Natl Acad. Sci. USA 111, 11037-11042 (2014).

53. Schamel, W. W. \& Reth, M. Monomeric and oligomeric complexes of the B cell antigen receptor. Immunity 13, 5-14 (2000).

54. Freeman, S. A. et al. Toll-like receptor ligands sensitize B-cell receptor signalling by reducing actin-dependent spatial confinement of the receptor. Nat. Commun. 6, 6168 (2015)

55. Bian, X., Kim, C. \& Karniadakis, G. E. 111 years of Brownian motion. Soft Matter 12, 6331-6346 (2016).

56. Pace, K. E., Lee, C., Stewart, P. L. \& Baum, L. G. Restricted receptor segregation into membrane microdomains occurs on human $\mathrm{T}$ cells during apoptosis induced by galectin-1. J. Immunol. 163, 3801-3811 (1999).

57. Stillman, B. N. et al. Galectin-3 and galectin-1 bind distinct cell surface glycoprotein receptors to induce T cell death. J. Immunol. 176, 778-789 (2006).

58. Symons, A., Cooper, D. N. \& Barclay, A. N. Characterization of the interaction between galectin-1 and lymphocyte glycoproteins CD45 and Thy-1. Glycobiology 10, 559-563 (2000).

59. Clark, M. C. et al. Galectin-3 binds to CD45 on diffuse large B-cell lymphoma cells to regulate susceptibility to cell death. Blood 120, 4635-4644 (2012).

60. Lin, J., Brown, V. K. \& Justement, L. B. Regulation of basal tyrosine phosphorylation of the $\mathrm{B}$ cell antigen receptor complex by the protein tyrosine phosphatase, CD45. J. Immunol. 149, 3182-3190 (1992).

61. Pani, G., Siminovitch, K. A. \& Paige, C. J. The motheaten mutation rescues B cell signaling and development in CD45-deficient mice. J. Exp. Med. 186, 581-588 (1997).

62. Heng, T. S. \& Painter, M. W. The Immunological Genome Project: networks of gene expression in immune cells. Nat. Immunol. 9, 1091-1094 (2008).

63. Gieseke, F. et al. Proinflammatory stimuli induce galectin-9 in human mesenchymal stromal cells to suppress T-cell proliferation. Eur. J. Immunol. 43, 2741-2749 (2013).

64. Chagan-Yasutan, H. et al. Galectin-9 plasma levels reflect adverse hematological and immunological features in acute dengue virus infection. $J$ Clin. Virol. 58, 635-640 (2013).

65. McSharry, B. P. et al. Human cytomegalovirus upregulates expression of the lectin galectin 9 via induction of beta interferon. J. Virol. 88, 10990-10994 (2014).

66. Tandon, R. et al. Galectin-9 is rapidly released during acute HIV-1 infection and remains sustained at high levels despite viral suppression even in elite controllers. AIDS Res. Hum. Retrovir. 30, 654-664 (2014).

67. Carrasco, Y. R., Fleire, S. J., Cameron, T., Dustin, M. L. \& Batista, F. D. LFA-1/ ICAM-1 interaction lowers the threshold of B cell activation by facilitating B cell adhesion and synapse formation. Immunity 20, 589-599 (2004). 
68. Liu, G. et al. Using ProHits to store, annotate, and analyze affinity purification-mass spectrometry (AP-MS) data. Curr. Protoc. Bioinformatics doi: https://doi.org/10.1002/0471250953.bi0816s39 (2012).

69. Crocker, J. C. \& Grier, D. G. Methods of digital video microscopy for colloidal studies. J. Colloid Interface Sci. 179, 289-310 (1996).

70. Ovesny, M., Krizek, P., Borkovec, J., Svindrych, Z. \& Hagen, G. M. ThunderSTORM: a comprehensive ImageJ plug-in for PALM and STORM data analysis and super-resolution imaging. Bioinformatics 30, 2389-2390 (2014).

\section{Acknowledgements}

We thank Kiera Clayton and Mario Ostrowski for human rGal9 ${ }^{33}$; Jose Grespan Guerios for help with dSTORM acquisition; Enayat Mohammad Gul for help with pull-down assays; Wen Zheng for help with cryosectioning and immunostaining of lymph nodes; and Jerry Gu for preparation of schematic model. This work was supported by funding from the Canadian Institutes of Health Research (CIHR; MOP-136808), Natural Sciences and Engineering Council of Canada (NSERC; RGPIN 418756-12), and Canada Research Chair (CRC; 905-231134) to B.T.; King Abdullah Scholarship to N.A.; NSERC graduate student fellowship to L.W.; and NSERC Undergraduate Student Research Award (USRA) to H.F. and W.Z.

\section{Author contributions}

A.C., N.A. and B.T. designed the research study. A.C., N.A., F.H.M.B., L.W., L.K.S. A.T.Q., Z.H., H.F. and B.T. conducted the experiments and data analysis. M.S. and D.M. O. provided input and assistance on dSTORM analysis. A.T.Q. and A.S. performed mass spectrometry analysis. A.C., N.A., F.H.M.B., L.W., L.K.S. and B.T. wrote the manuscript.

\section{Additional information}

Supplementary Information accompanies this paper at https://doi.org/10.1038/s41467018-05771-8.

Competing interests: The authors declare no competing interests.

Reprints and permission information is available online at http://npg.nature.com/ reprintsandpermissions/

Publisher's note: Springer Nature remains neutral with regard to jurisdictional claims in published maps and institutional affiliations.

(c) Open Access This article is licensed under a Creative Commons Attribution 4.0 International License, which permits use, sharing, adaptation, distribution and reproduction in any medium or format, as long as you give appropriate credit to the original author(s) and the source, provide a link to the Creative Commons license, and indicate if changes were made. The images or other third party material in this article are included in the article's Creative Commons license, unless indicated otherwise in a credit line to the material. If material is not included in the article's Creative Commons license and your intended use is not permitted by statutory regulation or exceeds the permitted use, you will need to obtain permission directly from the copyright holder. To view a copy of this license, visit http://creativecommons.org/ licenses/by/4.0/.

(C) The Author(s) 2018 